\title{
Konsensusempfehlungen der DRG/DGK/DGPK zum Einsatz der Herz- bildgebung mit Computertomografie und Magnetresonanztomografie
}

Consensus recommendations of the German Radiology Society (DRG), the German Cardiac Society (DGK) and the German Society for Pediatric Cardiology (DGPK) on the Use of Cardiac Imaging with Computed Tomography and Magnetic Resonance Imaging

für die AG Herz- und Gefäßdiagnostik der Deutschen Röntgengesellschaft

2 im Auftrag der Klinischen Kommission der Deutschen Gesellschaft für Kardiologie - Herz- und Kreislaufforschung

3 im Auftrag der Deutschen Gesellschaft für Pädiatrische Kardiologie

Dieses Konsensuspapier wurde in den Zeitschriften „Der Kardiologe“ 02/2012 und Röfo 04/2012 publiziert.

\section{Bibliografie}

DOI http://dx.doi.org/ 10.1055/s-0031-1299400

Online-Publikation: 2012 Fortschr Röntgenstr 2012; 184: 345-368 ๑ Georg Thieme Verlag KG Stuttgart · New York . ISSN 1438-9029

Korrespondenzadresse Prof. Dr. R. Fischbach Chefarzt, Radiologie, Neuroradiologie, und Nuklearmedizin, Asklepios Klinik Altona

Paul-Ehrlich-Str. 22763 Hamburg Tel.: ++49/40/18 1881-18 11 Fax: $++49 / 40 / 181881-4917$ r.fischbach@asklepios.com

\section{Zusammenfassung}

\section{$\nabla$}

Die kardiale Schnittbilddiagnostik mit der Magnetresonanztomografie (MRT) und der Computertomografie (CT) hat sich in der letzten Dekade technisch rasant weiterentwickelt. Diese Verbesserungen und die breite Verfügbarkeit moderner CT- und MRT-Systeme haben dazu geführt, dass beide Verfahren jetzt regelmäßig in der klinischen Routine eingesetzt werden. Dieses deutsche Konsensuspapier wurde daher gemeinsam von der Deutschen Gesellschaft für Kardiologie - Herz- und Kreislaufforschung, der Deutschen Röntgengesellschaft und der Deutschen Gesellschaft für Pädiatrische Kardiologie erarbeitet und orientiert sich nicht an Modalitäten und Methoden, sondern gliedert sich nach großen Krankheitsgruppen. Behandelt werden die koronare Herzerkrankung, Kardiomyopathien, Herzrhythmusstörungen, Klappenvitien, Perikarderkrankungen, erworbene und strukturelle Veränderungen sowie angeborene Herzfehler. Für unterschiedliche klinische Szenarien werden die beiden Schnittbildmodalitäten CT und MRT vergleichend gegenübergestellt und beiden Methoden in einem kurzen Textfeld bewertet.

\section{Einleitung \\ $\nabla$}

Die kardiale Schnittbilddiagnostik mit der Magnetresonanztomografie (MRT) und der Computertomografie (CT) hat sich in der letzten Dekade technisch rasant weiterentwickelt. In der CT sind in erster Linie eine Erhöhung der Anzahl der Detektorzeilen und damit eine größere Abdeckung sowie eine Verkürzung der Rotationszeiten zu nennen. In der MRT gab es zahlreiche neue Sequenzentwicklungen, die die Bildqualität deutlich verbessert haben, und auch der Einsatz von 3Tesla-Systemen liefert bei einigen Anwendungen

\section{Abstract \\ $\nabla$}

Cardiac magnetic resonance imaging (MRI) and computed tomography (CT) have been developed rapidly in the last decade. Technical improvements and broad availability of modern CT and MRI scanners have led to an increasing and regular use of both diagnostic methods in clinical routine. Therefore, this German consensus document has been developed in collaboration by the German Cardiac Society, German Radiology Society, and the German Society for Pediatric Cardiology. It is not oriented on modalities and methods, but rather on disease entities. This consensus document deals with coronary artery disease, cardiomyopathies, arrhythmias, valvular diseases, pericardial diseases and structural changes, as well as with congenital heart defects. For different clinical scenarios both imaging modalities CT and MRI are compared and evaluated in the specific context.

klare Vorteile. Neben der Bildqualität konnte auch die Robustheit der Verfahren deutlich verbessert werden, sodass jetzt auch bei schwierigen Untersuchungsbedingungen zuverlässig diagnostische Bilder erzeugt werden können.

Die beschriebenen Verbesserungen und die breite Verfügbarkeit moderner CT- und MRT-Systeme haben dazu geführt, dass beide Verfahren jetzt regelmäßig von Radiologen und Kardiologen in der klinischen Routine eingesetzt werden. Dieses Konsensuspapier wurde daher gemeinsam von der Deutschen Gesellschaft für Kardiologie Herz- und Kreislaufforschung, der Deutschen 
Röntgengesellschaft und der Deutschen Gesellschaft für Pädiatrische Kardiologie erarbeitet.

\section{Organisation des Redaktionskomitees}

Nach einem konstituierenden Treffen von Vertretern der Deutschen Gesellschaft für Kardiologie - Herz- und Kreislaufforschung und der Deutschen Röntgengesellschaft im Dezember 2008 wurden von beiden Fachgesellschaften Experten in der kardialen Schnittbilddiagnostik in das Redaktionskomitee entsandt. Um auch das Thema angeborene Herzfehler umfassend darzustellen, wurde das Redaktionskomitee anschließend durch Vertreter der Deutschen Gesellschaft für Pädiatrische Kardiologie ergänzt. Das Dokument wurden jeweils in Kleingruppen, in denen immer Kardiologen und Radiologen vertreten waren, erarbeitet und dann im Umlaufverfahren sowie bei einem Treffen im Frühjahr 2010 im gesamten Redaktionskomitee diskutiert.

Die vorliegenden Konsensusempfehlungen wurden von der Deutschen Röntgengesellschaft, der Deutschen Gesellschaft für Kardiologie - Herz- und Kreislaufforschung und der Deutschen Gesellschaft für Pädiatrische Kardiologie erarbeitet und genehmigt.

\section{Struktur der Empfehlungen \\ $\nabla$}

Die Struktur der Empfehlungen unterscheidet sich von den bisherigen deutsch- und englischsprachigen Publikationen zu diesem Thema. Die aktuelle Konsensusempfehlung orientiert sich nicht an Modalitäten und Methoden, sondern gliedert sich nach großen Krankheitsgruppen. Behandelt werden die koronare Herzerkrankung, Kardiomyopathien, Herzrhythmusstörungen, Klappenvitien, Perikarderkrankungen, erworbene und strukturelle Veränderungen sowie angeborene Herzfehler. Für unterschiedliche klinische Szenarien (asymptomatische Patienten, symptomatische Patienten, Zustand nach Therapie) werden dann die beiden Schnittbildmodalitäten CT und MRT vergleichend gegenübergestellt. Für jedes klinische Szenario wird die Aussagekraft der kardialen CT- und MRT-Diagnostik in einem kurzen Textfeld bewertet. Ergänzt wird diese Bewertung durch eine Zusammenstellung der wichtigsten Literaturstellen zum Thema. Die Indikation wurde anschließend im Redaktionskomitee im Konsensusverfahren auf einer 5-stufigen Skala bewertet. Dabei entspricht:

I1 Zuverlässig einsetzbar und anderen Verfahren überlegen I2 Diagnostische Genauigkeit vergleichbar mit anderen Verfahren I3 Einsatz technisch möglich und validiert, Indikation aber nur in Einzelfällen gegeben

U unklare Indikation, keine oder nicht kongruente Studienergebnisse

$\mathbf{K}$ keine Indikation

Falls keine aussagekräftigen Studien zu einzelnen Fragestellungen bzw. klinischen Szenarien vorlagen, entspricht die Empfehlung der Expertenmeinung des Redaktionskomitees.

\section{Gliederung}

$\nabla$

1. Koronare Herzerkrankung

1.1. Asymptomatische Individuen - Risikoabschätzung

1.2. Symptomatische Patienten - Nachweis von Stenosen

1.3. Bekannte Koronare Herzerkrankung - Therapieplanung

1.4. Status nach Koronarrevaskularisation - asymptomatisch

1.5. Status nach Koronarrevaskularisation - symptomatisch

2. Myokarderkrankungen

2.1. Kardial asymptomatische Individuen

2.2. Kardial symptomatische Individuen

2.3. Bekannte Myokarderkrankung - Therapieplanung

2.4. Status nach Behandlung einer Myokarderkrankung asymptomatisch

2.5. Status nach Behandlung einer Myokarderkrankung symptomatisch

3. Herzrhythmusstörungen

3.1. Asymptomatische Individuen

3.2. Symptomatische Individuen

3.3. Bekannte Arrhythmie - Therapieplanung

3.4. Status nach Behandlung einer Rhythmusstörung asymptomatisch

3.5. Status nach Behandlung einer Rhythmusstörung symptomatisch

4. Klappenvitien

4.1. Asymptomatische Individuen

4.2. Symptomatische Individuen - Nachweis einer Herzklappenerkrankung

4.3. Bekannte Herzklappenerkrankung - Therapieplanung

4.4. Status nach Klappenersatz/-intervention - asymptomatisch

4.5. Status nach Klappenersatz/-intervention - symptomatisch

5. Perikarderkrankungen

5.1. Asymptomatische Individuen

5.2. Symptomatische Individuen

5.3. Bekannte Perikarderkrankung - Therapieplanung

5.4. Status nach Perikarderkrankung - asymptomatisch

5.5. Status nach Perikarderkrankung - symptomatisch

6. Raumforderungen und Implantate (Erworbene strukturelle Veränderung am Herzen)

6.1. Asymptomatische Individuen

6.2. Symptomatische Individuen

6.3. Erworbene strukturelle Veränderung am Herzen - Therapieplanung

6.4.Z.n. Therapie einer erworbenen strukturellen Veränderung am Herzen - asymptomatisch

6.5.Z. n. Therapie einer erworbenen strukturellen Veränderung am Herzen - symptomatisch

7. Angeborene Herzerkrankungen

7.1. Asymptomatische Individuen

7.2. Symptomatische Individuen/Therapieplanung

7.3.Z. n. Therapie einer erworbenen strukturellen Herzerkrankung (symptomatisch/asymptomatisch) 
Tab. 1

Fragestellung

Bewertung MR

Bewertung CT

1. Koronare Herzerkrankung

1.1. Risikoabschätzung asymptomatischer Individuen

\begin{tabular}{|ll|}
\hline 1.1.1. Screening & $\begin{array}{l}\mathbf{K} \\
\text { Keine Indikation zur MRT }\end{array}$ \\
\hline 1.1.2. Stratifizierung nach Bestim- & $\mathbf{K}$ \\
\hline
\end{tabular}

K

mung der Risikofaktoren Keine Indikation zur MRT

Keine Indikation zur CT

13

Indikation zum koronaren Kalknachweis mittels Computertomografie als mögliche weitere Risikostratifikation bei Patienten mit einem intermediären KHK-Risiko (10 - $20 \%$ Ereignisrisiko in den nächsten 10 Jahren gemäß Framingham). Zahlreiche Studien zur prognostischen Bedeutung des Koronarkalknachweises mittels Computertomografie und zur Überlegenheit im Vergleich zu traditionellen Risikofaktoren. $[1-7]$.

Keine Indikation zum Kalknachweis bei hohem oder niedrigem KHK-Risiko.

K

Keine Indikation zur CT-Angiografie der Koronararterien.

1.1.3. Risikostratifizierung vor nicht koronarer Herzoperation zum Ausschluss von Koronarstenosen

\section{U}

Ein Ischämienachweis mittels Stress-MR liefert prognostische Informationen. Es liegen keine spezifischen Daten zu dieser Patientenpopulation vor.

\subsubsection{Risikostratifizierung vor nicht} kardialer Operation

\section{3}

Die Dobutamin-Stress MR kann zur präoperativen Risikostratifizierung eingesetzt werden.

$[13,14]$.

\section{2}

Wenn klinisch erforderlich, kann die CT-Angiografie der Koronararterien Koronarstenosen vor nicht koronaren Herzoperationen ausschließen. Mehrere Studien zum hohen negativ-prädiktiven Wert der koronaren CT-Angiografie bei niedriger Vortestwahrscheinlichkeit und zur diagnostischen Aussagekraft der CT-Angiografie bei Patienten vor nicht koronarer Herzoperation liegen vor.

[8-12].

\section{K}

Keine Indikation zur CT.

1.2. Nachweis signifikanter Steno-

sen bei symptomatischen Patienten

1.2.1. Stabile Angina Pectoris

1.2.1.1. Erstdiagnostik

1.2.1.2. Nach Ischämienachweis

\section{2}

Pharmakologische MR-Belastungsuntersuchung (Adenosin/ Dobutamin) zum Ausschluss ischämierelevanter Koronarstenosen bei intermediärer Vortestwahrscheinlichkeit, wenn ansonsten eine invasive Koronarangiografie erforderlich wäre (z. B. Ischämietest nicht möglich oder unzureichende Aussagekraft der Echokardiografie).

MRT der Single-Photonen-Emissions-ComputerTomografie in der Ischämiediagnostik überlegen. Keine Indikation für MR-Koronarangiografie. [15-24].

\section{K}

Bei validem Ischämienachweis im Vortest liefert die MR-Belastungsuntersuchung keine Zusatzinformationen

Keine Indikation für MR-Koronarangiografie.

[34].
12

CT-Angiografie zum Ausschluss von Koronarterienstenosen bei intermediärer Vortestwahrscheinlichkeit, wenn ansonsten eine invasive Koronarangiografie erforderlich wäre (z. B. Ischämietest nicht möglich). Mehrere Studien zum hohen negativ-prädiktiven Wert der koronaren CT-Angiografie bei niedriger bis mittlerer Vortestwahrscheinlichkeit, Nachweis einer sehr niedrigen klinischen Ereignisrate nach Ausschluss von Koronararterienstenosen mittels CT-Angiografie. $[8-10,25-33]$.

13

CT-Angiografie zum Ausschluss von Koronarterienstenosen, wenn der Ischämienachweis unklar bleibt oder im Widerspruch zur klinischen Einschätzung steht.

Mehrere Studien zum hohen negativ-prädiktiven Wert der koronaren CT-Angiografie, Nachweis einer niedrigen klinischen Ereignisrate nach Ausschluss von Koronararterienstenosen mittels CT-Angiografie auch bei Patienten mit pathologischem Ischämienachweis. $[8-10,25,32,33,35-37]$. 


\section{Tab. 1 (Fortsetzung)}

\section{Fragestellung}

1.2.2. Akutes Koronarsyndrom

1.2.2.1. Instabile Angina/Thoraxschmerz ohne EKG-Veränderungen und ohne Troponin-Anstieg

\subsubsection{NSTEMI}

\subsubsection{STEMI}

1.2.2.4. Differenzialdiagnose des akuten Koronarsyndroms 1.2.2.4.1. Myokarditis

\subsection{Aortendissektion}

1.2.2.4.3. Lungenarterienembolie

\section{kung - Therapieplanung}

1.3.1. Ischämiediagnostik: Patient mit Koronarstenose unklarer Relevanz

\section{Bewertung MR}

13

In der Akutphase, bei Beschwerdepersistenz oder Hochrisikokonstellation für KHK keine MR-Indikation. Pharmakologische MR-Belastungsuntersuchung (Adenosin/Dobutamin) zum Ausschluss ischämierelevanter Koronarstenosen bei stabilisierten Patienten ohne Hochrisikokonstellation.

Keine Indikation für MR-Koronarangiografie.

$$
[15,21-24,38-40] \text {. }
$$

I2

Bei Verdacht auf NSTEMI MRT ggf. zur Differenzialdiagnose (Myokarditis, Takotsubo). [49-53].

\section{U}

Risikostratifizierung (Infarktausmaß, Ödem, mikrovaskuläre Obstruktion).

$[54,55]$.

13

In der Frühphase nach STEMI ggf. MRT zur Risikostratifizierung (Infarktausmaß, mikrovaskuläre Obstruktion, Myocardial Salvage)

[56-65].

11

Nach Ausschluss eines ACS ist die MRT die bildgebende Methode der ersten Wahl zum Nachweis/Ausschluss einer Myokarditis als Differenzialdiagnose des ACS (siehe auch 2.2.2.5)

$[52,53,66]$.

12

MRA zum Nachweis/Ausschluss einer Aortendissektion in der Akutphase diagnostisch gleichwertig mit der $\mathrm{CT}$. Aufgrund der längeren Untersuchungsdauer und der schlechteren Überwachungsmöglichkeiten nur für stabile Patienten geeignet.

In der chronischen Phase Indikation zur Verlaufsbeurteilung und Therapieplanung als Alternative zur CT. $[67,68]$.

\section{3}

MR-Angiografie ist prinzipiell zum Nachweis einer Lungenarterienembolie geeignet. Aufgrund der längeren Untersuchungsdauer und der schlechteren Überwachungsmöglichkeiten nur für stabile Patienten geeignet.

In der chronischen Phase kann die MRA zur Verlaufsbeurteilung und Therapieplanung als Alternative zur CT eingesetzt werden.

$[71,72]$.

\section{Bewertung CT}

12

CT-Angiografie zum Ausschluss von Koronararterienstenosen bei klinisch niedriger bis intermediärer Vortestwahrscheinlichkeit für das Vorliegen eines akuten Koronarsyndroms geeignet.

[25, $41-48]$.

13

Bei Verdacht auf NSTEMI ggf. CT zum Ausschluss von Koronarstenosen indiziert.

$[25,41-48]$.

Keine Indikation zur CT

Ggf. CT-Angiografie zum Ausschluss von Koronararterienstenosen.

11

CT-Angiografie ist die Methode der Wahl. [68-70].

\section{1}

CT-Angiografie ist die Methode der Wahl. $[73,74]$.

\section{K}

Die Adenosin Stress-Perfusion kann funktionell signiKeine Indikation zur CT. fikante Stenosen mit hoher Genauigkeit identifizieren. Prognostische Daten liegen vor.

12

Die Dobutamin-Stress-Wandbewegungsanalyse kann funktionell signifikante Stenosen mit hoher Genauigkeit identifizieren. Prognostische Daten liegen vor. $[19,20-24,75-82]$. 


\section{Tab. 1 (Fortsetzung)}

\section{Fragestellung}

1.3.2. Vitalität
1.3.3. Durchführungsplanung der operativen oder interventionellen Revaskularisation

\section{Bewertung MR}

Die MRT ermöglicht bei Patienten mit eingeschränkter linksventrikulärer Funktion die Abschätzung der Wahrscheinlichkeit einer Funktionsverbesserung nach Revaskularisation.

I1

"Delayed Enhancement Imaging“ ist die bevorzugte Methode zur Vitalitätsdiagnostik.

12

Low-dose-Wandbewegungsstress mit Dobutamin kann zur funktionellen Vitalitätsbestimmung eingesetzt werden.

13

Bei Patienten mit intermediärer Transmuralität des Delayed Enhancement erbringt die Kombination der beiden Methoden zusätzliche Information. [83-92].

\section{K}

Keine Indikation zur MR-Angiografie für die technische Planung der Revaskularisation.

\section{Bewertung CT}

Keine Indikation zur CT.
Indikation zur CT-Angiografie zur Planung der interventionellen Rekanalisation von chronischen Verschlüssen der Koronararterien, Indikation zur CT-Angiografie der Anatomie aortokoronarer Bypassgefäße vor Re-Operation.

Mehrere Studien zur Aussagekraft der CT-Angiografie bezüglich des Erfolgs einer interventionellen Revaskularisation chronischer Koronararterienverschlüsse, Studien zum Nutzen der Darstellung der Anatomie von Bypassgefäßen bei der Planung kardialer Reoperationen.

[93-98].
1.4. Status nach Koronarrevaskularisation - asymptomatisch

1.4.1. Status nach $\mathrm{PCI}$ U Die MRT-Ischämiediagnostik kann zur Prognoseeinschätzung eingesetzt werden. $[19,20-24,75-82]$.

K

Die MR-Angiografie zur Darstellung der Koronararterien ist nicht sinnvoll. $[75,76,99-104]$.

1.4.2. Status nach aortokoronarem Bypass

U

Die MRT-Ischämiediagnostik kann zur Prognoseeinschätzung eingesetzt werden.

Keine Daten.

K

Die MR-Angiografie zur Darstellung der Bypässe und der Koronararterien ist nicht sinnvoll.

Keine Daten.

1.5. Status nach Koronarrevaskularisation - symptomatisch 1.5.1. Status nach $\mathrm{PCI}$

\section{1}

MRT mit Perfusion in Kombination mit Delayed Enhancement mit der Frage nach Ischämie und zur Prognosebestimmung. [105].

I1

Indikation zur Dobutamin-Stress-MRT mit der Frage nach Ischämie und zur Prognosebestimmung. $[19,20-24,75-82]$.

K

Die MR-Angiografie Zur Darstellung der Koronargefäße ist nicht sinnvoll.

[102 - 104, 106, 107].

$[18-20,75,77,108-118]$.
Keine Indikation zur CT.
Keine Indikation zur CT.

\section{U}

In bisherigen Studien ist die CT-Angiografie zur Beurteilung von Restenosen in Koronarstents nicht ausreichend zuverlässig. 


\section{Tab. 1 (Fortsetzung)}

\section{Fragestellung}

1.5.2. Status nach aortokoronarem Bypass

1.6. Koronararterielle Anomalien

\section{Bewertung MR}

I1

MRT mit Perfusion in Kombination mit Delayed Enhancement mit der Frage nach Ischämie und zur Prognosebestimmung.

[77, 91, 105, 108, 110, 112].

12

Indikation zur Dobutamin-Stress-MRT mit der Frage nach Ischämie und zur Prognosebestimmung.

$[18,75,116,118]$.

13

Direkte Darstellung der Bypässe bei der Frage Bypassverschluss oder Bypassstenose. MRT in der Genauigkeit der Bypassdarstellung der CT unterlegen.

[119-126].

\section{2}

MR-Koronarangiografie ist eine der Methoden der Wahl.

[132-135].

\section{Bewertung CT}

13

Indikation zur CT-Angiografie, wenn lediglich der Nachweis der Bypass-Offenheit erforderlich ist (z. B. Bypass in der invasiven Angiografie nicht darstellbar). Die Darstellung der nativen Koronargefäße ist nicht indiziert. Zahlreiche Studien zur hohen Sensitivität und Spezifität der CT-Angiografie bezüglich des Nachweises von Verschlüssen und Stenosen von aortokoronaren Bypassgefäßen.

[127-131].

\section{1}

CT-Angiografie ist eine der Methoden der Wahl. Zahlreiche Studien zur Wertigkeit der CT-Angiografie für die Charakterisierung von Koronaranomalien.

[136-140].

\section{Myokarderkrankungen}

2.1. Kardial asymptomatische Indi-

viduen

2.1.1. Kardiale Beteiligung bei Systemerkrankungen (z. B. Sarkoidose; Amyloidose; Hämochromatose; Sklerodermie)

\subsubsection{Myokarditis}

\section{1}

Indikation zur Klärung der Myokardbeteiligung. Bestimmung der ventrikulären Funktion, Myokardmasse.

[141-149].

\section{I1}

Nachweis von entzündungsbedingten Myokardveränderungen, Bestimmung der ventrikulären Funktion, Differenzialdiagnosen.

\section{K}

Keine Indikation zur CT.

Keine Indikation zur CT.

$$
[51,150] \text {. }
$$

\subsection{Kardial symptomatische Indivi-} duen

2.2.1 Untersuchung der links- und rechtsventrikulären Funktion

2.2.2. Nachweis und Differenzialdiagnose einer Kardiomyopathie oder entzündlichen Herzerkrankung

\section{I1}

Die MRT wird als Referenzmethode für die Untersuchung der links- und rechtsventrikulären Funktion angesehen. $[148,149]$.

\section{1}

Indikation zur Festlegung der Diagnose und des Schweregrads der Erkrankung anhand morphologischer und funktioneller Parameter. Prognostische Bedeutung der Untersuchung (Myokardmasse, Kontrastmittelaufnahme). [151-153].

\subsubsection{Hypertrophe Kardiomyopa-} thie

2.2.2.2. Dilatative Kardiomyopathie

\section{1}

In Ergänzung zur Basisdiagnostik Indikation zur Festlegung der Diagnose und des Schweregrads der Erkrankung anhand morphologischer und funktioneller Parameter.

Prognostische Bedeutung der Untersuchung (Myokardmasse, Kontrastmittelaufnahme) $[159,160]$.

\section{I1}

In Ergänzung zur Basisdiagnostik Indikation zur Festlegung der Diagnose und des Schweregrads der Erkrankung anhand morphologischer und funktioneller Parameter. Nachweis oder Ausschluss von LV-Thromben. Prognostische Bedeutung der Untersuchung (Myokardmasse, Kontrastmittelaufnahme). [161-163].
Keine Indikation zur CT.

\section{U}

Indikation zur CT-Angiografie als Alternative zur Echokardiografie und Magnetresonanztomografie nur, wenn beide Verfahren nicht die gewünschte diagnostische Aussage (Myokardhypertrophie und Funktion) liefern.

Zahlreiche Studien belegen eine exzellente Übereinstimmung der CT-Funktionsanalyse mit MRT, Echokardiografie und SPECT. [154-158].

U

2.2.2. 


\section{Tab. 1 (Fortsetzung)}

\section{Fragestellung}

2.2.2.3. Restriktive Kardiomyopathie

2.2.2.4. Non-Compaction-Kardiomyopathie

2.2.2.5. Arrhythmogene rechtsventrikuläre Kardiomyopathie (ARVC)

2.2.2.6. Takotsubo-Kardiomyopathie

2.2.7. Myokarditis

2.2.2.8. Löffler-Endokarditis und andere nicht bakterielle Endokarditiden

2.2.2.9. Nachweis von anderen Myokarderkrankungen

\section{Bewertung MR}

I1

Indikation zur Festlegung der Diagnose und des Schweregrads der Erkrankung anhand morphologischer und funktioneller Parameter.

Abgrenzung gegenüber der Pericarditis constrictiva durch die Perikardbeurteilung.

Prognostische Bedeutung der Untersuchung (Myokardmasse, Kontrastmittelaufnahme).

[164 - 167].

11

In Ergänzung zur Basisdiagnostik Indikation zur Festlegung der Diagnose und des Schweregrads der Erkrankung anhand morphologischer und funktioneller Parameter.

$[168,169]$.

11

In Ergänzung zur Basisdiagnostik Indikation zur Festlegung der Diagnose und des Schweregrads der Erkrankung anhand morphologischer und funktioneller Parameter.

$[170,171]$

I1

In Ergänzung zur Basisdiagnostik Indikation zur Festlegung der Diagnose und des Schweregrads der Erkrankung anhand morphologischer und funktioneller Parameter. $[50,66,172]$.

\section{1}

Methode der Wahl. Indikation zur Festlegung der Diagnose und des Schweregrads der Erkrankung anhand morphologischer und funktioneller Parameter, insbesondere aber auch anhand der fokalen T2-Signalintensität und Kontrastmittelaufnahme. [173-177].

11

Bei Embolisationen im Rahmen einer entzündlichen Endokarderkrankung sollte ein MRT durchgeführt werden. Diese dient einerseits dem Nachweis von Thromben und dem direkten Nachweis inflammatorischer Endokardregionen.

[178].

13

MRT gilt neben der Myokardbiopsie als Referenzstandard zum Nachweis struktureller Myokardveränderungen.

$[179,180]$

\section{Bewertung CT}

U

2.2.2.

U

2.2.2.

2.2.2.

K

Keine Indikation zur CT.

\section{K}

Keine Indikation zur CT.

K

Keine Indikation zur CT.

K

Keine Indikation zur CT.

\subsubsection{Nachweis einer Dyssynchro-} nie

\section{U}

Derzeit keine validen Daten. 181, 182].

I1

Ausmaß und Lokalisation der Narbe kann Vorhersage für Response nach CRT geben.

[188-192].

\section{3}

Indikation zur MRT als Alternative zur Echokardiografie.

[193-195].
I1

Indikation zur CT-Angiografie als Methode der Wahl. [183-187].

Es liegen keine Daten vor.

U

Indikation zur CT-Angiografie als Alternative zur Echokardiografie und Magnetresonanztomografie für die Darstellung der LV-Funktion und Dyssynchronie nur, wenn beide Verfahren nicht die gewünschte diagnostische Aussage liefern.

Ggf. Kombination mit der Darstellung von Koronarvenen zur Identifikation des Zielgefäßes. [196]. 


\section{Tab. 1 (Fortsetzung)}

\section{Fragestellung}

2.4. Status nach Behandlung einer Myokarderkrankung - asymptomatisch

\subsection{Status nach Behandlung einer} Myokarderkrankung - symptomatisch

\section{Bewertung MR}

12

In Ergänzung zur Basisdiagnostik Verlaufskontrolle morphologischer und funktioneller Parameter in Abhängigkeit von der klinischen Konsequenz. Prognostischer Stellenwert der Untersuchung (Funktionsparameter, Kontrastmittelaufnahme). $[172,197,198]$.

\section{2}

Verlaufskontrolle morphologischer und funktioneller Parameter in Abhängigkeit von der klinischen Konsequenz.

Prognostischer Stellenwert der Untersuchung (Funktionsparameter, Kontrastmittelaufnahme).

[153].

\section{Herzrhythmusstörungen}

\subsection{Asymptomatische Individuen}

3.2. Symptomatische Individuen
Keine Indikation zur MRT.

\section{2}

Indikation zur kardialen MRT bei Patienten ohne klare Diagnose nach Abschluss der Basisdiagnostik. Zahlreiche Studien belegen die Bedeutung der MRT zum Nachweis struktureller Herzerkrankungen.

$[170,199,200]$.

\subsection{Bekannte Rhythmusstörung -}

Therapieplanung

\subsubsection{Anatomische Referenz vor} Ablation

\section{3}

Einige aktuelle Studien belegen die Möglichkeiten der MRT zur anatomischen Venen- und Vorhofdarstellung vor Ablation.

[201-203].

\section{Bewertung CT}

K

Keine Indikation zur CT.
U

Indikation zur CT des Herzens als Alternative zur Echokardiografie und Magnetresonanztomografie nur, wenn beide Verfahren nicht die gewünschte diagnostische Aussage liefern.

\subsection{Status nach Behandlung einer} Rhythmusstörung - asymptomatisch

3.5. Status nach Behandlung einer

Rhythmusstörung - symptomatisch

\subsubsection{Pulmonalvenenstenose nach} Ablation

\section{Klappenvitien}

\subsection{Asymptomatische Individuen}

4.1.1 Screening

4.2. Nachweis einer Herzklappenerkrankung bei symptomatischen Patienten

4.2.1. Klappenstenose und -insuffizienz

4.2.1.1. Aortenklappenstenose

\section{3}

MRT ist Alternative zu TTE/TEE.

Planimetrie der Öffnungsfläche meistens gut möglich. $[216,217]$

\subsubsection{Aortenklappeninsuffizienz}

MRT ermöglicht die Quantifizierung des Regurgitationsvolumens mittels Flussmessung. MRT ermöglicht außerdem eine Beurteilung einer möglichen konsekutiven LV-Vergrößerung und eine exakte Quantifizierung der LV Funktion. [202-225].
12

CT zur Darstellung der Anatomie von Vorhöfen und Pulmonalvenen. Mehrere Studien bestätigen die Wertigkeit der CT als anatomische Referenz mit und ohne Bildfusion in der Intervention. [204 - 208].

\section{K}

Keine Indikation zur CT.
12

CT-Angiografie ist geeignet zur Darstellung der Pulmonalvenen und zur Detektion von Pulmonalvenenstenosen nach Katheterablation.

$[214,215]$
Keine Indikation zur MRT.
Keine Indikation zur CT.
13

Planimetrie der Klappenöffnungsfläche mittels CTAngiografie als Alternative zur Echokardiografie und Magnetresonanztomografie möglich - nur, wenn beide Verfahren nicht die gewünschte diagnostische Aussage liefern.

[218-221].

Keine Indikation zur CT. 


\section{Tab. 1 (Fortsetzung)}

\section{Fragestellung}

4.2.1.3. Mitralklappenstenose

4.2.1.5. Pulmonalklappenstenose

4.2.1.6. Pulmonalklappeninsuffizienz

4.2.1.7. Trikuspidalklappe
4.2.1.4. Mitralklappeninsuffizienz

\section{Bewertung MR}

\section{3}

Ergänzend zur Echokardiografie kann mittels MRT der Schweregrad ermittelt werden. [226].

\section{3}

Ergänzend zur Echokardiografie kann mittels MRT der Schweregrad ermittelt werden.

$[222,223,227]$.

12

Ergänzend zur Echokardiografie kann mittels MRT der Schweregrad ermittelt werden.

$[228,229]$.

11

Ergänzend zur Echokardiografie kann mittels MRT der Schweregrad ermittelt werden.

$[230,231]$.

12

Ergänzend zur Echokardiografie kann mittels MRT der Schweregrad ermittelt werden.

Sehr gute Beurteilung der Morphologie und anatomischer Varianten, z. B. Ebstein-Anomalie.

[232].

\subsubsection{Endokarditis}

4.2.2.1. Nativklappen

\subsubsection{Klappenprothesen}

4.3. Bekannte Herzklappenerkran-

kung - Therapieplanung

4.3.1. Ausschluss Koronarstenosen

Keine Indikation zur MRT.
Vegetationen sind nicht sicher zu erkennen.

U

Wenn klinisch erforderlich, kann die MRT zur Diagnostik eines paravalvulären Abszesses eingesetzt werden. [233].

\section{K}

Vegetationen sind nicht sicher zu erkennen.

\section{U}

Wenn klinisch erforderlich, kann die MRT zur Diagnostik einer paravalvulären Leckage/eines Abszesses ein-

[236]. gesetzt werden.

\section{Bewertung CT}

K

Keine Indikation zur CT.

K

Keine Indikation zur CT.

K

Keine Indikation zur CT.

\section{K}

Keine Indikation zur CT.

K

Keine Indikation zur CT.

Vegetationen sind nicht sicher zu erkennen.

I3

Wenn erforderlich, kann die CT mit hoher Genauigkeit zur Diagnostik eines paravalvulären Abszesses eingesetzt werden.

[234, 235].

K

Vegetationen sind nicht sicher zu erkennen.

13

Wenn erforderlich, kann die CT mit hoher Genauigkeit zur Diagnostik eines paravalvulären Abszesses eingesetzt werden.

[234, 235].
4.3.3. Planung perkutaner Klappeninterventionen

4.3.3.1. Aortenklappe

4.3.3.2. Mitralklappe

\section{K}

Keine Daten.
Wenn klinisch erforderlich, kann die CT-Angiografie der Koronararterien Koronarstenosen vor nicht koronaren Herzoperationen ausschließen. Mehrere Studien zum hohen negativ-prädiktiven Wert der koronaren CT-Angiografie bei niedriger Vortest-Wahrscheinlichkeit und auch bei Patienten vor nicht koronarer kardialer Operation.

$[11,12,237]$.

\section{U}

$\mathrm{CT}$ in Einzelfällen mit spezieller Fragestellung gerechtfertigt, z. B. Ausmaß der Verkalkung der Aorta ascendens.

\section{1}

Die MRT mit der Möglichkeit der 3-D-Darstellung ermöglicht die Vermessung des Bulbus und Beurteilung der Aorta im ges. Verlauf, inkl. des peripheren Zugangswegs. [238].

U

Die MRT erlaubt die anatomische Darstellung der Mitralklappe und Quantifizierung der Insuffizienz. [241].
CT-Angiografie ist Methode der Wahl. Mehrere SingleCenter.Studien zur Wertigkeit der CT zur Planung und Kontrolle des perkutanen Aortenklappenersatzes. $[235,239,240]$.

U

Die CT erlaubt die Darstellung der Anatomie und Geometrie der Mitralklappe.

[242]. 


\section{Tab. 1 (Fortsetzung)}

\section{Fragestellung}

4.4. Status nach Behandlung einer Herzklappenerkrankung - asymptomatisch

4.5. Status nach Behandlung einer Herzklappenerkrankung - symptomatisch
4.5.1 Klappenfunktion

U

Planimetrie der Klappenöffnungsfläche von Bioprothesen mittels MRT möglich, wenn die Echokardiografie nicht die gewünschte diagnostische Aussage liefert.

\subsubsection{Leckagen, Abszesse}

\section{U}

Wenn klinisch erforderlich kann die MRT zur Diagnostik einer paravalvulären Leckage/eines Abszesses eingesetzt werden.

$[233,236]$.

\section{Bewertung CT}

K

Keine Indikation zur CT.
Planimetrie der Klappenöffnungsfläche mittels CTAngiografie bei Bioprothesen als Alternative zur Echokardiografie und Magnetresonanztomografie möglich - nur, wenn beide Verfahren nicht die gewünschte diagnostische Aussage liefern.

13

Die CT kann mit hoher Genauigkeit zur Diagnostik einer paravalvulären Leckage/eines Abszesses eingesetzt werden.

$[234,235]$.

\section{K}

Keine Indikation zur CT.
5.2. Ausschluss/Nachweis einer Perikarderkrankung bei symptomatischen Individuen

\subsubsection{Perikarderguss}

Keine Indikation zur MRT.

\section{3}

\subsection{Perikarderkrankung - Therapie-} planung

5.3.1. Ausschluss von begleitenden Koronarstenosen

Keine Indikation zur MRT

\section{K}

kardektomie

Keine Indikation zur MRT.

\subsection{Status nach Therapie einer Peri-} karderkrankung - asymptomatisch

5.5. Status nach Therapie einer Perikarderkrankung - symptomatisch
Das Ausmaß eines Perikardergusses, mögliche Ursachen und die funktionelle Relevanz können bestimmt werden.

$[243,244]$.

Eine perikardiale Entzündung und ggf. eine myokardiale Mitreaktion können mit der MRT zuverlässig dargestellt werden.

[246-249].

Die MRT ermöglich die direkte Beurteilung des Perikards und die umfassende Beurteilung der rechts- und linksventrikulären Funktion. Differenzialdiagnose zur restriktiven Kardiomyopathie.

[164, 165, $250-253]$.
Das Ausmaß eines Perikardergusses kann zuverlässig bestimmt werden. Die Unterscheidung von hämorrhagischen und serösen Ergüssen ist möglich. [245].

\section{K}

Keine Indikation zur CT.

12

Gute Methode zur Darstellung von Perikardverkalkung.

Mögliche Methode zum Nachweis einer Verdickung des Perikards.

$[254,255]$.

\section{3}

Nach der operativen Behandlung einer Perikarderkrankung ermöglicht die MRT die Beurteilung der Funktionsverbesserung und den Ausschluss eines Rezidivs nach Tumorresektionen. [258-260].

\section{I1}

Nach der operativen Behandlung einer Perikarderkrankung ermöglicht die MRT die Beurteilung der Funktionsverbesserung und den Ausschluss eines Rezidivs nach Tumorresektionen.
Wenn klinisch erforderlich, kann die CT-Angiografie der Koronararterien Koronarstenosen vor nicht koronaren Herzoperationen ausschließen.

13

Indikation zur CT für die Abbildung des verkalkten Perikards, zur Erleichterung der Resektions- und Zugangsplanung. [254, 256, 257].

\section{K}

Keine Indikation zur CT.
Im Einzelfall zur Beurteilung des Resektionsergebnisses.

[258-264]. 


\section{Tab. 1 (Fortsetzung)}

\section{Fragestellung \\ 6. Raumforderungen \& Implantate \\ (Erworbene strukturelle Verände-}

Bewertung MR

Bewertung CT

rung am Herzen)

6.1. Asymptomatische Individuen

6.1.1. Infiltration des Herzens bei

Malignomen benachbarter Struktu-

I1

Zuverlässige Beurteilung einer Infiltration von Perikard oder Myokard.

[265-269].

\section{3}

Indikation zur CT als Alternative und Ergänzung zur Echokardiografie und Magnetresonanztomografie, wenn beide Verfahren nicht die gewünschte diagnostische Aussage liefern.

[270-273].

\subsubsection{Kardiale Implantate}

6.1.2.1. Lage von Schrittmacher/

ICD-Elektroden

K

Keine Indikation zur MRT.

K

Keine Indikation zur MRT.

(s. 6.2.3.2).

\subsection{Symptomatische Individuen}

6.2.1. Kardiale Raumforderung als Emboliequelle

6.2.1.1. Vorhofthromben

U

Die MRT erlaubt den Nachweis von Vorhofthromben, die Aussagekraft ist eingeschränkt (Vorhofohr).

[273, 274].

6.2.1.2. Ventrikelthromben

6.2.1.3. Kardiale Tumoren

6.2.2. Abklärung echokardiografisch nachgewiesener Raumforderungen

\section{I1}

Die MRT ist der Echokardiografie beim Nachweis ventrikulärer Thromben überlegen.

Vorteile bestehen im Nachweis apikaler oder kleiner wandständiger Thromben. [280-284].

I1

Die MRT erlaubt die zuverlässige Darstellung und Differenzierung von Tumoren. Eingeschränkte Aussagekraft bei Tumoren der Herzklappen.

[270, 272, 285-288].

\section{1}

Die MRT liefert wertvolle Zusatzinformationen bezüglich Größenausdehnung, topografischen und anatomischen Beziehungen, Gewebecharakterisierung und zur möglichen Differenzierung zwischen benignen und malignen Prozessen.

[266, 272 287, 288].

6.2.3. Verdacht auf Dislokation ode Fehlfunktion von kardialen Implantaten

6.2.3.1. Lage von Schrittmacher/ ICD Elektroden

K

Keine Indikation zur MRT.

\section{U}

Beurteilung des Restshunts möglich. [291-295].

Keine Indikation zur MRT.
6.2.3.3. Klappenprothesen
Keine Indikation zur CT.

Keine Indikation zur CT.
CT (2 Phasen) bei Kontraindikationen oder unklarer Aussage der TEE.

Mehrere Studien zeigen hohe Sensitivität, aber eingeschränkte Spezifität zur Detektion von linksatrialen Thromben durch $\mathrm{CT}$. [275-279].

Indikation zur CT als Alternative zur Echokardiografie und Magnetresonanztomografie - nur, wenn beide Verfahren nicht die gewünschte diagnostische Aussage liefern.

\section{3}

Indikation zur CT als Alternative und als Ergänzung zur Echokardiografie und Magnetresonanztomografie, wenn beide Verfahren nicht die gewünschte diagnostische Aussage liefern.

[270-273].

\section{3}

Indikation zur CT als Alternative und als Ergänzung zur Magnetresonanztomografie, wenn diese nicht möglich ist oder nicht die gewünschte diagnostische Aussage liefert.

[270-272].

11

CT als Ergänzung zur konventionellen Röntgendiagnostik. Zwei Studien zeigen die Überlegenheit der CT gegenüber anderen verfügbaren Bildgebungsmodalitäten für diese Fragestellung. $[289,290]$.

U

CT als Ergänzung zur konventionellen Röntgendiagnostik.

U

CT als Ergänzung zur konventionellen Röntgendiagnostik.

6.3. Bekannte erworbene struktu-

relle Veränderungen am Herzen -

Therapieplanung 
Tab. 1 (Fortsetzung)

\section{Fragestellung}

6.3.1. OP-Planung

\section{Bewertung MR}

I1

Die kardiale MRT stellt bei benignen und malignen kardialen Tumoren hinsichtlich der Frage Operabilität/ präoperative Planung eine Methode der Wahl dar. [270].

\subsection{Zustand nach Therapie einer} strukturellen Veränderung am Herzen - asymptomatisch

\subsection{Zustand nach Therapie einer} strukturellen Veränderung am Herzen - symptomatisch

\section{Angeborene Herzerkrankungen}

\subsection{Asymptomatische Individuen}

7.1.1. Unklare RA/RV-Vergrößerung/Hypertrophie

- z. B. Vorhofseptumdefekt

- Myokardiale Genese

- Klappenvitien (s. Kapitel 4.2.3. und 4.2.4.)

\section{2}

Eine Indikation für die kardiale MRT ergibt sich bei eingeschränkter Aussagekraft der Echokardiografie, diskrepanten bzw. unklaren Befunden. Keine systematischen Studien.

\section{2}

Eine Indikation für die kardiale MRT ergibt sich bei eingeschränkter Aussagekraft der Echokardiografie, diskrepanten bzw. unklaren Befunden. Keine systematischen Studien.

\section{2}

Echokardiografie ist Methode der ersten Wahl; bei nicht oder nicht vollständig beurteilbaren Veränderungen ergänzende MRT sinnvoll.

[170, 296, 297].

\section{Bewertung CT}

\section{3}

Indikation zur CT als Alternative und Ergänzung zur Echokardiografie und Magnetresonanztomografie, wenn beide Verfahren nicht die gewünschte diagnostische Aussage liefern.

[270].

\section{K}

Keine Indikation zur CT.

U

Indikation zur CT als Alternative und Ergänzung zur Echokardiografie und Magnetresonanztomografie, wenn beide Verfahren nicht die gewünschte diagnostische Aussage liefern.

\subsubsection{Unklare LA/LV-Vergrößerung/} Hypertrophie (mit/ohne art. Hypertonus)

- z. B. durch Volumenlast (Shunts durch Koronarfisteln, Angiome)

- myokardiale durch ventrikuläre Drucklast

- Klappenvitien (s. Kapitel 4.2.1. und 4.2.2.)

7.2. Symptomatische Individuen/

Therapieplanung

7.2.1. Anomalien des Situs/der Zirkulation

\section{2}

Zahlreiche Studien, die den Vorteil der MRT-Volumetrie und Funktionsanalyse des LA und LV gegenüber der Echokardiografie hervorheben, insbesondere bei pathologischer Ventrikelgeometrie.

[296, 304, 305].

\section{3}

Alternative und/oder Ergänzung zur, Echokardiografie, Angiografie und MRT, wenn diese nicht die gewünschte diagnostische Aussage liefern oder bei MRTKontraindikationen vorliegen.

Zahlreiche in der Regel retrospektive Studien, die die Wertigkeit der CT zur Visualisierung insbesondere bei Neugeborenen und Kleinkindern zeigen.

[298-303].

(siehe 7.1.1.)

\section{I1}

Überlegenheit der MRT gegenüber der Echokardiografie und Herzkatheteruntersuchung in zahlreichen, teils prospektiven Studien, insbesondere bei der Darstellung der pulmonalvenösen und systemvenösen Verbindungen und ihren Beziehungen zu mediastinalen Strukturen. [306-308].

\section{3}

(siehe 7.1.1.).

CT mit aktueller Technik (niedrige Dosis, sehr kurze Scanzeit) kann als Ersatz für Katheterangiografie und als Alternative zur MRT wegen deutlich kürzerer Untersuchungszeit gewertet werden.

12

Zur Notfalldiagnostik.

[309-316].

\subsubsection{Anomalien der Vorhöfe und} der Venen

7.2.2.1. Vorhofseptumdefekte

7.2.2.2. Lungenvenenfehlmündungen

\section{2}

Mehrere, teilweise prospektive Studien, zeigen gegenüber der Echokardiografie bei atypischen Defekten eine bessere Korrelation zur tatsächlichen Defektgröße. Zuverlässige nicht invasive Bestimmung der Shuntgröße.

[317-322].

11

Zuverlässige native oder kontrastmittelgestützte Visualisierung, und Quantifizierung des Links-RechtsShunts mittels der Volumetrie und Flussmessung. [324-326].

\section{U}

Keine Studien bei Kindern, bisher lediglich Case Reports und Erfahrungsberichte. Mehrere, gute Korrelationen zur tatsächlichen Defektgröße gegenüber der Echokardiografie, insbesondere bei atypischen Defekten. Keine Shuntbestimmung möglich. [323].

12

Zuverlässige und schnelle Darstellung der Lungenvenen insbesondere bei Neugeborenen, Säuglingen und kritisch kranken Kindern mit vertretbarer Strahlenexposition als Alternative zur MRT und Herzkatheter möglich.

[298, 300, 301]. 
Tab. 1 (Fortsetzung)

\begin{tabular}{|c|c|c|}
\hline Fragestellung & Bewertung MR & Bewertung CT \\
\hline $\begin{array}{l}\text { 7.2.2.3. Systemvenenfehlmündun- } \\
\text { gen }\end{array}$ & $\begin{array}{l}\text { 12 } \\
\text { Die Darstellung der Systemvenen ist zuverlässig mit- } \\
\text { tels MRT möglich, wenn sie nicht mit der Echokardio- } \\
\text { grafie gelingt. } \\
\text { [327-330]. }\end{array}$ & $\begin{array}{l}\mathbf{1 2} \\
\text { (siehe 7.1.1.). }\end{array}$ \\
\hline \multicolumn{3}{|l|}{ 7.2.3. Anomalien der AV-Klappen } \\
\hline 7.2.3.1. Morbus Ebstein & $\begin{array}{l}\text { I2 } \\
\text { Nur wenige Studien. Die Objektivierung der rechts- } \\
\text { und linksventrikulären Größe und Funktion werden als } \\
\text { wertvoll eingeschätzt. } \\
\text { [331-333]. }\end{array}$ & $\begin{array}{l}\mathbf{U} \\
\text { (siehe 7.1.1.). }\end{array}$ \\
\hline $\begin{array}{l}\text { 7.2.3.2. Atrioventrikuläre Septum- } \\
\text { defekte }\end{array}$ & $\begin{array}{l}13 \\
\text { Nur wenige Studien bei atrioventrikulären Septumde- } \\
\text { fekten im Säuglingsalter, da meist echokardiografisch } \\
\text { beurteilbar. Sowohl die Volumetrie der Ventrikel als } \\
\text { auch die Bestimmung der Shuntgröße und Shuntrich- } \\
\text { tung sind zuverlässig möglich. } \\
{[334-336] \text {. }}\end{array}$ & $\begin{array}{l}\mathbf{U} \\
\text { (siehe 7.2.2.1.). }\end{array}$ \\
\hline \multicolumn{3}{|l|}{$\begin{array}{l}\text { 7.2.4. Anomalien der Ventrikel/ } \\
\text { Ventrikelsepten }\end{array}$} \\
\hline $\begin{array}{l}\text { 7.2.4.1. Ventrikelaneurysma/-diver- } \\
\text { tikel }\end{array}$ & $\begin{array}{l}12 \\
\text { Die Darstellung kongenitaler Divertikel und Aneurys- } \\
\text { men wird in zahlreichen Reviews vor allem aufgrund } \\
\text { der Vitalitätsdiagnostik als wertvoll eingeschätzt. } \\
\text { Thromben können zuverlässiger als mit der Echokar- } \\
\text { diografie nachgewiesen werden. } \\
\text { [337-339]. }\end{array}$ & $\begin{array}{l}\mathbf{U} \\
\text { (siehe 7.1.1.). }\end{array}$ \\
\hline 7.2.4.2. VSD mit komplexen Vitium & $\begin{array}{l}\text { I2 } \\
\text { Zahlreiche Studien und Reviews belegen den Nutzen } \\
\text { bei der Therapieplanung komplexer Vitien, mit ge- } \\
\text { genüber der Echokardiografie wichtigen Zusatz-Infor- } \\
\text { mationen. } \\
{[340-349] \text {. }}\end{array}$ & $\begin{array}{l}\mathbf{U} \\
\text { (siehe 7.2.2.1.). }\end{array}$ \\
\hline \multicolumn{3}{|l|}{$\begin{array}{l}\text { 7.2.5. Anomalien der Semilunar- } \\
\text { klappen }\end{array}$} \\
\hline $\begin{array}{l}\text { 7.2.5.1. Pulmonalstenose/-insuffi- } \\
\text { zienz }\end{array}$ & $\begin{array}{l}\text { I1 } \\
\text { Zahlreiche Studien zeigen den Nutzen der kardialen } \\
\text { MRT beim Timing von Pulmonalklappenersatzproze- } \\
\text { duren in Hinblick auf ein ventrikuläres Remodelling. } \\
{[231,350-355] \text {. }}\end{array}$ & $\begin{array}{l}\text { I3 } \\
\text { Planimetrie der Klappenöffnungsfläche mittels CT- } \\
\text { Angiografie als Alternative zur Echokardiografie und } \\
\text { MRT möglich - nur, wenn beide Verfahren nicht die } \\
\text { gewünschte diagnostische Aussage liefern. } \\
{[218-221] \text {. }}\end{array}$ \\
\hline $\begin{array}{l}\text { 7.2.5.2. Aortenstenose/-insuffi- } \\
\text { zienz }\end{array}$ & $\begin{array}{l}\text { I3 } \\
\text { Die Aortenklappe ist in der Regel echokardiografisch } \\
\text { gut beurteilbar. Bei unklaren Befunden kann eine er- } \\
\text { gänzende MRT hilfreich sein. }\end{array}$ & $\begin{array}{l}\text { I3 } \\
\text { (siehe 7.2.5.1.). }\end{array}$ \\
\hline 7.2.5.3. Sinus/valsalva/Aneurysma & $\begin{array}{l}12 \\
\text { Insbesondere wenn zusätzliche Malformationen vor- } \\
\text { liegen, ist die MRT durch die dreidimensionale Dar- } \\
\text { stellung wertvoll. } \\
\text { [356-359]. }\end{array}$ & $\begin{array}{l}\text { I3 } \\
\text { Zahlreiche Case Reports und einzelne Reviews. Alter- } \\
\text { native zur MRT. } \\
{[360] \text {. }}\end{array}$ \\
\hline $\begin{array}{l}\text { 7.2.6. Anomalien der großen Gefäße } \\
\text { 7.2.6.1. Malposition; Ring-Sling } \\
\text { 7.2.6.2. Isthmusstenose; Bogen- } \\
\text { hypoplasien; Divertikel } \\
\text { 7.2.6.3. Ductus mit Verdacht auf } \\
\text { PHT } \\
\text { 7.2.6.4. Pulmonalarterienstenose/ } \\
\text { Aplasie } \\
\text { 7.2.6.5. Truncus arteriosus } \\
\text { 7.2.6.6. MAPCAS; veno-venöse } \\
\text { Kollateralen }\end{array}$ & $\begin{array}{l}12 \\
\text { Eine Vielzahl von Studien und Reviews zeigt den Stel- } \\
\text { lenwert der kardialen MRT zur Darstellung der extra- } \\
\text { kardialen Gefäße in der Therapieplanung. } \\
\text { [300, } 361-371] \text {. }\end{array}$ & $\begin{array}{l}\text { I2 } \\
\text { Indikation zur CT als Alternative und/oder Ergänzung } \\
\text { zur invasiven Angiografie, Echokardiografie und MRT, } \\
\text { wenn diese Verfahren nicht die gewünschte diagnos- } \\
\text { tische Aussage liefern. Eine Vielzahl von Studien und } \\
\text { Reviews zeigt den Stellenwert der kardialen CT zur } \\
\text { Darstellung der extrakardialen Gefäße und des Tra- } \\
\text { cheobronchialbaums in der Therapieplanung. } \\
\text { [298-302, 310, 372, 373]. }\end{array}$ \\
\hline
\end{tabular}


Tab. 1 (Fortsetzung)

\section{Fragestellung}

7.2.6.7. Koronararterien

- Ursprungsanomalien (siehe

Kap. 1.6.)

- Fisteln, Angiome

- Kawasaki, andere Vaskulitiden

Evaluierung für Ross-, Switch-Operation

7.2.6.8. Vaskulär bedingte Atemwegsstenosen bzw. unklare Stridor-। Schluckbeschwerden bei vaskulärem Ring, Sling oder Divertikel

7.3. Zustand nach Therapie einer

angeborenen Herzerkrankung (symptomatisch/asymptomatisch)

7.3.1. Single-ventricle Palliationen

- Nativer Zustand: Protected PA oder PHT

- Glenn/Hemi-Fontan

- Fontan-Palliation

\subsubsection{Operationen und Interventio-} nen mit biventrikulärer Korrektur 7.3.2.1. „Einfache“ Septal-Defekte und Lungenvenen-Fehlmündungen (ASD; VSD; AVSD; PAPVR; PDA; AOPA Kollateralen)

7.3.2.2. Rekonstruktion der RV-Pulmonalis-Kontinuität \pm VSD-Patchverschluss (Fallot, PA-VSD, DORV \& subaortalem VSD, TAC I-III, intracavitäre RV-Stenose)

7.3.2.3. Arterieller Switch \pm VSDVerschluss (d-TGA \pm VSD; DORV mit subpulmonalem VSD; CC-TGA und VSD ohne LVOTO)

7.3.2.4. „Atrial redirection”: Baffle nach Senning/Mustard

- d-TGA $\pm V S D$, Double-Switch bei CC-TGA

7.3.2.5. Re-Konnektion des LV mit der transponierten Aorta: RastelliOperation (DORV; TGA/VSD/PS)

7.3.2.6. Erkrankungen der thorakalen Aorta (Stenosen; Anomalien)

\section{Bewertung MR}

12

Die MR-Koronarangiografie kann die proximalen Koronargefäßverläufe und die anatomischen Lagebeziehungen zu den großen thorakalen Gefäßen/kardialen Strukturen zuverlässig beurteilen. Ggfs. in Kombination mit Dobutamin-Stress-MR-Wandbewegungsanalyse zum Nachweis eines ischämierelevanten anomalen Koronarverlaufs. Größere koronararterielle Fisteln (arteriovenös, ventrikulär) können dargestellt werden.

[132, 374].

12

Zahlreiche retrospektive Studien und Case Reports belegen die Wertigkeit zur Visualisierung vaskulärer Varianten und Pathologien bei Stridor und Schluckbeschwerden.

[377].

\section{Bewertung CT}

12

Die CT-Angiografie ist eine der Methoden der Wahl. Zahlreiche Studien zur Wertigkeit der CT/Angiografie für die Charakterisierung von Koronaranomalien.

[136-140, 375, 376].

12

Zahlreiche retrospektive Studien belegen die Wertigkeit der CT zur Visualisierung vaskulärer Varianten und Pathologien bei Stridor und Schluckbeschwerden. [298, 300, 301].

\section{1}

Die MR-Volumetrie ist beim univentrikulären Herzen anderen Methoden überlegen. Zusätzlich kann die pulmonale Durchblutung zuverlässig beurteilt werden.
[378-380].

Die CT hilft bei der Beurteilung ventrikulärer Volumina und der Funktion und erlaubt die Darstellung der Ventrikelgeometrie. Bisher keine Studien bei Kindern, lediglich Case Reports und Erfahrungsberichte. [314].

13

Die MRT erlaubt neben der morphologischen Beurteilung und der Volumetrie die Bestimmung von Qp:Qs mit hoher Genauigkeit und kann eine invasive Untersuchung ersetzen.

[317, 381, 382].

11

Die MRT erlaubt die Quantifizierung einer PK-Insuffizienz mit hoher Genauigkeit und ist Methode der Wahl zur Verlaufsbeurteilung. RVOT und Pulmonalarterien lassen sich zuverlässig beurteilen. [230, 329, 383-385].

\section{I1}

Die MRT ermöglicht eine zuverlässige Darstellung der Pulmonalarterien und der proximalen Koronararterien. Der postoperative Einsatz nach Koronar-Reimplantation ist durch einzelne Studien belegt.

Die ergänzende Adenosin-Stress-MRT stellt eine alternative Methode der Ischämie-Diagnostik dar. [374, 386 - 389].

I1

Die kardiale MRT ermöglicht die Darstellung der postoperativen Situation mit hoher Genauigkeit; die Sensitivität für kleine Lecks ist eingeschränkt. [343, 389-391].

I1

Die Darstellung der postoperativen anatomischen Situation ist mittels MRT mit hoher Genauigkeit möglich.

[343].

\section{2}

Die MRT ermöglicht eine zuverlässige Darstellung der Aorta und kann viele postoperative Fragestellungen beantworten.

[392-395].

(siehe 7.1.1.).
Indikation zur CT als Alternative zur Echokardiografie und MRT, wenn diese Verfahren nicht die gewünschte diagnostische Aussage liefern oder wenn MRT-Kontraindikationen vorliegen. Der RVOT lässt sich mit hoher Sicherheit beurteilen.

12

Insbesondere die postoperative Koronardarstellung gelingt mittels kardialer $\mathrm{CT}$ und ist gut belegt. [375].

\section{3}

Indikation zur CT als Alternative zur Echokardiografie und MRT, wenn diese Verfahren nicht die gewünschte diagnostische Aussage liefern oder wenn MRT-Kontraindikationen vorliegen.

13

(siehe 7.3.2.4.).

\section{2}

Alternative und/oder Ergänzung zur Angiografie, Echokardiografie und MRT, wenn diese nicht die gewünschte diagnostische Aussage liefern oder wenn MRT-Kontraindikationen vorliegen. Methode der Wahl nach Stentimplantation. 


\section{Redaktionskomitee}

Stephan Achenbach ${ }^{2}$, Jörg Barkhausen ${ }^{1}$, Roman Fischbach ${ }^{1}$, Matthias Gutberlet ${ }^{1}$, Samir Sarikouch ${ }^{3}$, Holger Thiele ${ }^{2}$

\section{Arbeitsgruppe CT}

Stephan Achenbach ${ }^{2}$, Roman Fischbach ${ }^{1}$, Martin Hoffmann ${ }^{1}$

\section{Arbeitsgruppe MRT}

Jörg Barkhausen ${ }^{1}$, Meinrad Beer ${ }^{1}$, Thorsten Dill ${ }^{2}$, Matthias Gutberlet $^{1}$, Armin Huber ${ }^{2}$, Peter Hunold ${ }^{1}$, Christoph Klein ${ }^{2}$, Gabriele Krombach $^{1}$, Karl-Friedrich Kreitner ${ }^{1}$, Joachim Lotz ${ }^{1}$, David Maintz $^{1}$, Heiko Marholdt ${ }^{2}$, Nico Merkle ${ }^{2}$, Daniel Messroghli², Stephan Miller ${ }^{1}$, Ingo Paetsch ${ }^{2}$, Peter Radke ${ }^{2}$, Henning Steen ${ }^{2}$, Holger Thiele $^{2}$

\section{Arbeitsgruppe angeborene Herzfehler}

Philipp Beerbaum ${ }^{3}$, Joachim Eichhorn ${ }^{3}$, Sohrab Fratz ${ }^{3}$, Matthias Gutberlet $^{1}$, Titus Kühne ${ }^{3}$, Joachim Lotz ${ }^{1}$, Samir Sarikouch ${ }^{3}$

\section{Literatur}

1 Detrano R, Guerci AD, Carr JJ et al. Coronary calcium as a predictor of coronary events in four racial or ethnic groups. N Engl J Med 2008; 358: $1336-1345$

2 Arad Y, Goodman KJ, Roth $M$ et al. Coronary calcification, coronary disease risk factors, C-reactive protein, and atherosclerotic cardiovascular disease events: the St. Francis Heart Study. J Am Coll Cardiol 2005; 46: $158-165$

3 Park R, Detrano R, Xiang $M$ et al. Combined use of computed tomography coronary calcium scores and C-reactive protein levels in predicting cardiovascular events in nondiabetic individuals. Circulation 2002; 106: 2073-2077

4 Greenland P, LaBree L, Azen SP et al. Coronary artery calcium score combined with Framingham score for risk prediction in asymptomatic individuals. Jama 2004; 291: 210-215

5 Taylor AJ, Bindeman J, Feuerstein I et al. Coronary calcium independently predicts incident premature coronary heart disease over measured cardiovascular risk factors: mean three-year outcomes in the Prospective Army Coronary Calcium (PACC) project. J Am Coll Cardiol 2005; 46: 807-814

6 Greenland P, Alpert JS, Beller GA et al. 2010 ACCF/AHA guideline for assessment of cardiovascular risk in asymptomatic adults: executive summary: a report of the American College of Cardiology Foundation/American Heart Association Task Force on Practice Guidelines. J Am Coll Cardiol 2010; 56: 2182-2199

7 Erbel R, Möhlenkamp S, Moebus S et al. Coronary risk stratification, discrimination, and reclassification improvement based on quantification of subclinical coronary atherosclerosis: the Heinz Nixdorf Recall study. J Am Coll Cardiol 2010; 56: 1397-1406

8 Meijboom WB, Meijs MF, SchuijfJD et al. Diagnostic accuracy of 64-slice computed tomography coronary angiography: a prospective, multicenter, multivendor study. J Am Coll Cardiol 2008; 52: 2135 - 2144

9 Budoff MJ, Dowe D, Jollis JG et al. Diagnostic performance of 64-multidetector row coronary computed tomographic angiography for evaluation of coronary artery stenosis in individuals without known coronary artery disease: results from the prospective multicenter ACCURACY (Assessment by Coronary Computed Tomographic Angiography of Individuals Undergoing Invasive Coronary Angiography) trial. J Am Coll Cardiol 2008; 52: 1724-1732

10 Mowatt G, Cook JA, Hillis GS et al. 64-Slice computed tomography angiography in the diagnosis and assessment of coronary artery disease: systematic review and meta-analysis. Heart 2008; 94: 1386-1393

11 Scheffel H, Leschka S, Plass A et al. Accuracy of 64-slice computed tomography for the preoperative detection of coronary artery disease in patients with chronic aortic regurgitation. Am J Cardiol 2007; 100: $701-776$

12 Catalan P, Leta R, Hidalgo A et al. Ruling out coronary artery disease with noninvasive coronary multidetector $\mathrm{CT}$ angiography before noncoronary cardiovascular surgery. Radiology 2011; 258: 426-434
13 Poldermans D, Bax JJ, Boersma E et al. Guidelines for pre-operative cardiac risk assessment and perioperative cardiac management in noncardiac surgery: the Task Force for Preoperative Cardiac Risk Assessment and Perioperative Cardiac Management in Non-cardiac Surgery of the European Society of Cardiology (ESC) and European Society of Anaesthesiology (ESA). Eur Heart J 2009; 30: 2769-2812

14 Rerkpattanapipat P, Morgan TM, Neagle CM et al. Assessment of preoperative cardiac risk with magnetic resonance imaging. Am J Cardiol 2002; 90: 416-419

15 Jahnke C, Nagel E, Gebker $R$ et al. Prognostic value of cardiac magnetic resonance stress tests. Adenosine stress perfusion and dobutamine stress wall motion imaging. Circulation 2007; 115: 1769-1776

16 Bernhardt P, Engels T, Levenson B et al. Prediction of necessity for coronary artery revascularization by adenosine contrast-enhanced magnetic resonance imaging. Int J Cardiol 2006; 112: 184-190

17 Costa MA, Shoemaker S, Futamatsu $\mathrm{H}$ et al. Quantitative magnetic resonance perfusion imaging detects anatomic and physiologic coronary artery disease as measured by coronary angiography and fractional flow reserve. J Am Coll Cardiol 2007; 50: 514-522

18 Gebker R, Jahnke C, Manka R et al. Additional value of myocardial perfusion imaging during dobutamine stress magnetic resonance for the assessment of coronary artery disease. Circ Cardiovasc Imaging 2008; 1: $122-130$

19 Paetsch I, Jahnke C, Wahl A et al. Comparison of dobutamine stress magnetic resonance, adenosine stress magnetic resonance, and adenosine stress magnetic resonance perfusion. Circulation 2004; 110: 835 842

20 Schwitter J, Wacker C, van Rossum A et al. MR-IMPACT: comparison of perfusion-cardiac magnetic resonance with single-photon emission computed tomography for the detection of coronary artery disease in a multicentre, multivendor, randomized trial. European Heart Journal 2008; 29: 480-489

21 Bodi V, Sanchis J, Lopez-Lereu MP et al. Prognostic value of dipyridamole stress cardiovascular magnetic resonance imaging in patients with known or suspected coronary artery disease. J Am Coll Cardiol 2007; 50: $1174-1179$

22 Korosoglou G, Elhmidi Y, Steen H et al. Prognostic value of high-dose dobutamine stress magnetic resonance imaging in 1,493 consecutive patients: Assessment of myocardial wall motion and perfusion. J Am Coll Cardiol 2010; 56: 1225-1234

23 Bodi V, Husser O, Sanchis J et al. Prognostic implications of dipyridamole cardiac MR imaging: a prospective multicenter registry. Radiology 2012; 262: 91 - 100

24 Greenwood JP, Maredia N, Younger JF et al. Cardiovascular magnetic resonance and single-photon emission computed tomography for diagnosis of coronary heart disease (CE-MARC): a prospective trial. Lancet 2011; 379: $453-460$

25 Meijboom WB, van Mieghem CA, Mollet NR et al. 64-slice computed tomography coronary angiography in patients with high, intermediate, or low pretest probability of significant coronary artery disease. J Am Coll Cardiol 2007; 50: 1469-1475

26 Hadamitzky M, Freissmuth B, Meyer T et al. Prognostic value of coronary computed tomographic angiography for prediction of cardiac events in patients with suspected coronary artery disease. JACC Cardiovasc Imaging 2009; 2: 404-411

27 Min JK, Shaw LJ, Berman DS et al. Costs and clinical outcomes in individuals without known coronary artery disease undergoing coronary computed tomographic angiography from an analysis of Medicare category III transaction codes. Am J Cardiol 2008; 102: 672-678

28 Ostrom MP, Gopal A, Ahmadi N et al. Mortality incidence and the severity of coronary atherosclerosis assessed by computed tomography angiography. J Am Coll Cardiol 2008; 52: 1335 -1343

29 Gopal A, Nasir K, Ahmadi N et al. Cardiac computed tomographic angiography in an outpatient setting: an analysis of clinical outcomes over a 40-month period. J Cardiovasc Comput Tomogr 2009; 3: 90 -95

30 Shaw LJ, Berman DS, Hendel RC et al. Prognosis by coronary computed tomographic angiography: matched comparison with myocardial perfusion single-photon emission computed tomography. J Cardiovasc Comput Tomogr 2008; 2: 93-101

31 van Werkhoven JM, Gaemperli O, Schuijf JD et al. Multislice computed tomography coronary angiography for risk stratification in patients with an intermediate pretest likelihood. Heart 2009; 95: 1607-1611

32 Shuman WP, May JM, Branch KR et al. Negative ECG-gated cardiac CT in patients with low-to-moderate risk chest pain in the emergency de- 
partment: 1-year follow-up. American journal of roentgenology 2010; 195: 923 - 927

33 Taylor AJ, Cerqueira M, Hodgson JM et al. ACCF/SCCT/ACR/AHA/ASE/ ASNC/NASCI/SCAI/SCMR 2010 appropriate use criteria for cardiac computed tomography. A report of the American College of Cardiology Foundation Appropriate Use Criteria Task Force, the Society of Cardiovascular Computed Tomography, the American College of Radiology, the American Heart Association, the American Society of Echocardiography, the American Society of Nuclear Cardiology, the North American Society for Cardiovascular Imaging, the Society for Cardiovascular Angiography and Interventions, and the Society for Cardiovascular Magnetic Resonance. J Am Coll Cardiol 2010; 56: 1864-1894

34 Nandalur KR, Dwamena BA, Choudhri AF et al. Diagnostic performance of stress cardiac magnetic resonance imaging in the detection of coronary artery disease: a meta-analysis. J Am Coll Cardiol 2007; 50: $1343-1353$

35 Hines JL, Danciu SC, Shah M et al. Use of multidetector computed tomography after mildly abnormal myocardial perfusion stress testing in a large single-specialty cardiology practice. J Cardiovasc Comput Tomogr 2008; 2: $372-378$

36 Danciu SC, Herrera CJ, Stecy PJ et al. Usefulness of multislice computed tomographic coronary angiography to identify patients with abnormal myocardial perfusion stress in whom diagnostic catheterization may be safely avoided. Am J Cardiol 2007; 100: 1605 - 1608

37 Abidov A, Gallagher MJ, Chinnaiyan KM et al. Clinical effectiveness of coronary computed tomographic angiography in the triage of patients to cardiac catheterization and revascularization after inconclusive stress testing: results of a 2-year prospective trial. J Nucl Cardiol 2009; 16: $701-713$

38 Cury RC, Shash K, Nagurney JT et al. Cardiac magnetic resonance with T2-weighted imaging improves detection of patients with acute coronary syndrome in the emergency department. Circulation 2008; 118 : $837-844$

39 Hombach V, Merkle N, Kestler HA et al. Characterization of patients with acute chest pain using cardiac magnetic resonance imaging. Clin Res Cardiol 2008; 97: 760-767

40 Lockie T, Nagel E, Redwood S et al. Use of cardiovascular magnetic resonance imaging in acute coronary syndromes. Circulation 2009; 119 : $1671-1681$

41 Hollander JE, Chang AM, Shofer FS et al. One-year outcomes following coronary computerized tomographic angiography for evaluation of emergency department patients with potential acute coronary syndrome. Acad Emerg Med 2009; 16: 693-698

42 Hollander JE, Chang AM, Shofer FS et al. Coronary computed tomographic angiography for rapid discharge of low-risk patients with potential acute coronary syndromes. Ann Emerg Med 2009; 53: 295 - 304

43 Goldstein JA, Gallagher MJ, O'Neill WW et al. A randomized controlled trial of multi-slice coronary computed tomography for evaluation of acute chest pain. J Am Coll Cardiol 2007; 49: 863-871

44 Hoffmann U, Nagurney JT, Moselewski F et al. Coronary multidetector computed tomography in the assessment of patients with acute chest pain. Circulation 2006; 114: $2251-2260$

45 Rubinshtein R, Halon DA, Gaspar T et al. Usefulness of 64-slice cardiac computed tomographic angiography for diagnosing acute coronary syndromes and predicting clinical outcome in emergency department patients with chest pain of uncertain origin. Circulation 2007; 115: $1762-1768$

46 Hoffmann U, Bamberg F, Chae CU et al. Coronary computed tomography angiography for early triage of patients with acute chest pain: the ROMICAT (Rule Out Myocardial Infarction using Computer Assisted Tomography) trial. J Am Coll Cardiol 2009; 53: 1642 - 1650

47 Chang SA, Choi SI, Choi EK et al. Usefulness of 64-slice multidetector computed tomography as an initial diagnostic approach in patients with acute chest pain. Am Heart J 2008; 156: 375 - 383

48 Goldstein JA, Chinnaiyan KM, Abidov A et al. The CT-STAT (Coronary Computed Tomographic Angiography for Systematic Triage of Acute Chest Pain Patients to Treatment) trial. J Am Coll Cardiol 2011; 58: $1414-1422$

49 Eitel I, Behrendt F, Schindler K et al. Differential diagnosis of the apical ballooning syndrome using contrast enhanced magnetic resonance imaging. Eur Heart J 2008; 29: 2651 - 2659

50 Eitel I, von Knobelsdorff-Brenkenhoff F, Bernhardt $P$ et al. Clinical characteristics and cardiovascular magnetic resonance findings in stress (Takotsubo) cardiomyopathy: A multicenter series in Europe and North America. JAMA 2011; 306: 277-286
51 Assomull RG, Lyne JC, Keenan $\mathrm{N}$ et al. The role of cardiovascular magnetic resonance in patients presenting with chest pain, raised troponin, and unobstructed coronary arteries. Eur Heart J 2007; 28: 1242 - 1249

52 Baccouche H, Mahrholdt $H$, Meinhardt $G$ et al. Diagnostic synergy of non-invasive cardiovascular magnetic resonance and invasive endomyocardial biopsy in troponin-positive patients without coronary artery disease. Eur Heart J 2009; 30: 2869 - 2879

53 Laissy JP, Hyafil F, Feldman LJ et al. Differentiating acute myocardial infarction from myocarditis: diagnostic value of early- and delayed-perfusion cardiac MR imaging. Radiology 2005; 237: 75-82

54 Mewton N, Bonnefoy E, Revel D et al. Presence and extent of cardiac magnetic resonance microvascular obstruction in reperfused non-STelevated myocardial infarction and correlation with infarct size and myocardial enzyme release. Cardiology 2009; 113: 50-58

55 Raman SV, Simonetti OP, Winner MWIII et al. Cardiac magnetic resonance with edema imaging identifies myocardium at risk and predicts worse outcome in patients with non-ST-segment elevation acute coronary syndrome. J Am Coll Cardiol 2010; 55: 2480-2488

56 Beek AM, Kuhl HP, Bondarenko 0 et al. Delayed contrast-enhanced magnetic resonance imaging for the prediction of regional functional improvement after acute myocardial infarction. J Am Coll Cardiol 2003; 42: 895-901

57 Bruder O, Breuckmann F, Jensen C et al. Prognostic impact of contrastenhanced CMR early after acute ST segment elevation myocardial infarction (STEMI) in a regional STEMI network: results of the „Herzinfarktverbund Essen“. Herz 2008; 33: 136-142

58 Gerber BL, Garot J, Bluemke DA et al. Accuracy of contrast-enhanced magnetic resonance imaging in predicting improvement of regional myocardial function in patients after acute myocardial infarction. Circulation 2002; 106: 1083-1089

59 Hombach V, Grebe O, Merkle $N$ et al. Sequelae of acute myocardial infarction regarding cardiac structure and function and their prognostic significance as assessed by magnetic resonance imaging. Eur Heart J 2005; 26: $549-557$

60 Nijveldt R, Beek AM, Hofman MB et al. Late gadolinium-enhanced cardiovascular magnetic resonance evaluation of infarct size and microvascular obstruction in optimally treated patients after acute myocardial infarction. J Cardiovasc Magn Reson 2007; 9: 765 - 770

61 Shapiro MD, Nieman K, Nasir K et al. Utility of cardiovascular magnetic resonance to predict left ventricular recovery after primary percutaneous coronary intervention for patients presenting with acute ST-segment elevation myocardial infarction. Am J Cardiol 2007; 100: 211 216

$62 W u$ E, Ortiz JT, Tejedor P et al. Infarct size by contrast enhanced cardiac magnetic resonance is a stronger predictor of outcomes than left ventricular ejection fraction or end-systolic volume index: prospective cohort study. Heart 2008; 94: 730-736

$63 \mathrm{Wu}$ KC, Zerhouni EA, Judd RM et al. Prognostic significance of microvascular obstruction by magnetic resonance imaging in patients with acute myocardial infarction. Circulation 1998; 97: 765-772

64 de Waha S, Desch S, Eitel I et al. Impact of early vs. late microvascular obstruction assessed by magnetic resonance imaging on long-term outcome after ST-elevation myocardial infarction: a comparison with traditional prognostic markers. Eur Heart J 2010; 31: 2660-2668

65 Eitel I, Desch S, Fuernau G et al. Prognostic significance and determinants of myocardial salvage assessed by cardiovascular magnetic resonance in acute reperfused myocardial infarction. J Am Coll Cardiol 2010; 55: 2470 - 2479

66 Eitel I, Behrendt F, Schindler K et al. Differential diagnosis of suspected apical ballooning syndrome using contrast-enhanced magnetic resonance imaging. Eur Heart J 2008; 29: 2651 - 2659

67 Nienaber CA, Kische S, Skriabina $V$ et al. Noninvasive imaging approaches to evaluate the patient with known or suspected aortic disease. Circ Cardiovasc Imaging 2009; 2: 499-506

68 Shiga T, Wajima Z, Apfel CC et al. Diagnostic accuracy of transesophageal echocardiography, helical computed tomography, and magnetic resonance imaging for suspected thoracic aortic dissection: systematic review and meta-analysis. Arch Intern Med 2006; 166: 1350-1356

69 Erbel R, Alfonso F, Boileau C et al. Diagnosis and management of aortic dissection. Eur Heart J 2001; 22: 1642 - 1681

70 Mammen L, Yucel E, Khan A et al. ACR Appropriateness Criteria ${ }^{\circledR}$ acute chest pain - suspected aortic dissection. Reston (VA): American College of Radiology (ACR) online publication: 2008, $1-6$

71 Haage $P$, Piroth $W$, Krombach $G$ et al. Pulmonary embolism: comparison of angiography with spiral computed tomography, magnetic reso- 
nance angiography, and real-time magnetic resonance imaging. Am J Respir Crit Care Med 2003; 167: 729-734

72 Kluge A, Luboldt W, Bachmann G. Acute pulmonary embolism to the subsegmental level: diagnostic accuracy of three MRI techniques compared with 16-MDCT. Am J Roentgenol 2006; 187: W7-W14

73 Remy-Jardin M, Pistolesi M, Goodman LR et al. Management of suspected acute pulmonary embolism in the era of CT angiography: a statement from the Fleischner Society. Radiology 2007; 245: 315-329

74 Stein PD, Woodard PK, Weg JG et al. Diagnostic pathways in acute pulmonary embolism: recommendations of the PIOPED II investigators. Am J Med 2006; 119: 1048 - 1055

75 Jahnke C, Nagel E, Gebker $R$ et al. Prognostic value of cardiac magnetic resonance stress tests: adenosine stress perfusion and dobutamine stress wall motion imaging. Circulation 2007; 115: 1769-1776

76 Wallace EL, Morgan TM, Walsh TF et al. Dobutamine cardiac magnetic resonance results predict cardiac prognosis in women with known or suspected ischemic heart disease. JACC Cardiovasc Imaging 2009; 2: 299-307

77 Bodi V, Sanchis J, Lopez-Lereu MP et al. Prognostic value of dipyridamole stress cardiovascular magnetic resonance imaging in patients with known or suspected coronary artery disease. J Am Coll Cardiol 2007; 50: $1174-1179$

78 Bodi V, Sanchis J, Lopez-Lereu MP et al. Prognostic and therapeutic implications of dipyridamole stress cardiovascular magnetic resonance on the basis of the ischaemic cascade. Heart 2009; 95: 49-55

79 Giang TH, Nanz D, Coulden R et al. Detection of coronary artery disease by magnetic resonance myocardial perfusion imaging with various contrast medium doses: first European multi-centre experience. Eur Heart J 2004; 25: 1657-1665

80 Tonino PA, De Bruyne B, Pijls NH et al. Fractional flow reserve versus angiography for guiding percutaneous coronary intervention. $\mathrm{N}$ Engl J Med 2009; 360: 213-224

81 Watkins S, McGeoch R, Lyne J et al. Validation of magnetic resonance myocardial perfusion imaging with fractional flow reserve for the detection of significant coronary heart disease. Circulation 2009; 120 : $2207-2213$

82 Wolff SD, Schwitter J, Coulden R et al. Myocardial first-pass perfusion magnetic resonance imaging: a multicenter dose-ranging study. Circulation 2004; 110: $732-737$

83 Kaandorp TA, Bax IJ, Schuijf JD et al. Head-to-head comparison between contrast-enhanced magnetic resonance imaging and dobutamine magnetic resonance imaging in men with ischemic cardiomyopathy. Am J Cardiol 2004; 93: 1461 - 1464

84 Wellnhofer E, Olariu A, Klein C et al. Magnetic resonance low-dose dobutamine test is superior to SCAR quantification for the prediction of functional recovery. Circulation 2004; 109: 2172 -2174

85 Baer FM, Voth E, Deutsch HJ et al. Predictive value of low dose dobutamine transesophageal echocardiography and fluorine- 18 fluorodeoxyglucose positron emission tomography for recovery of regional left ventricular function after successful revascularization. J Am Coll Cardiol 1996; 28: 60-69

86 Baer FM, Voth E, Schneider CA et al. Comparison of low-dose dobutamine-gradient-echo magnetic resonance imaging and positron emission tomography with [18F]fluorodeoxyglucose in patients with chronic coronary artery disease. A functional and morphological approach to the detection of residual myocardial viability. Circulation 1995; 91: $1006-1015$

87 Gutberlet M, Frohlich M, Mehl S et al. Myocardial viability assessment in patients with highly impaired left ventricular function: comparison of delayed enhancement, dobutamine stress MRI, end-diastolic wall thickness, and TI201-SPECT with functional recovery after revascularization. Eur Radiol 2005; 15: 872-880

88 Hunold P, Kreitner KF, Barkhausen J. [„Dead or alive?“: how and why myocardial viability imaging by cardiac MRI works]. Fortschr Röntgenstr 2007; 179: 1016-1024

89 Sandstede JJ, Bertsch G, Beer $M$ et al. Detection of myocardial viability by low-dose dobutamine Cine MR imaging. Magn Reson Imaging 1999; 17: $1437-1443$

$90 \mathrm{Kim} \mathrm{RJ,Wu} \mathrm{E,} \mathrm{Rafael} \mathrm{A} \mathrm{et} \mathrm{al.} \mathrm{The} \mathrm{use} \mathrm{of} \mathrm{contrast-enhanced} \mathrm{magnetic}$ resonance imaging to identify reversible myocardial dysfunction. N Engl J Med 2000; 343: 1445-1453

91 Klein C, Nagel E, Gebker R et al. Magnetic resonance adenosine perfusion imaging in patients after coronary artery bypass graft surgery. JACC Cardiovasc Imaging 2009; 2: 437-445
92 Kuhl HP, Beek AM, van der Weerdt AP et al. Myocardial viability in chronic ischemic heart disease: comparison of contrast-enhanced magnetic resonance imaging with (18)F-fluorodeoxyglucose positron emission tomography. J Am Coll Cardiol 2003; 41: 1341 - 1348

93 Soon $\mathrm{KH}$, Cox $N$, Wong A et al. CT coronary angiography predicts the outcome of percutaneous coronary intervention of chronic total occlusion. J Interv Cardiol 2007; 20: 359-366

94 Mollet NR, Hoye A, Lemos PA et al. Value of preprocedure multislice computed tomographic coronary angiography to predict the outcome of percutaneous recanalization of chronic total occlusions. Am J Cardiol 2005; 95: $240-243$

95 Gasparovic H, Rybicki FJ, Millstine J et al. Three dimensional computed tomographic imaging in planning the surgical approach for redo cardiac surgery after coronary revascularization. Eur J Cardiothorac Surg 2005; 28: $244-249$

96 Aviram G, Sharony R, Kramer A et al. Modification of surgical planning based on cardiac multidetector computed tomography in reoperative heart surgery. Ann Thorac Surg 2005; 79: 589-595

97 Kamdar AR, Meadows TA, Roselli EE et al. Multidetector computed tomographic angiography in planning of reoperative cardiothoracic surgery. Ann Thorac Surg 2008; 85: 1239-1245

98 Cho JR, Kim YJ, Ahn CM et al. Quantification of regional calcium burden in chronic total occlusion by 64-slice multi-detector computed tomography and procedural outcomes of percutaneous coronary intervention. Int J Cardiol 2010; 145: 9-14

99 Al-Saadi $N$, Nagel E, Gross $M$ et al. Improvement of myocardial perfusion reserve early after coronary intervention: assessment with cardiac magnetic resonance imaging. J Am Coll Cardiol 2000; 36: 1557- 1564

100 Fenchel M, Franow A, Stauder NI et al. Myocardial perfusion after angioplasty in patients suspected of having single-vessel coronary artery disease: improvement detected at rest-stress first-pass perfusion MR imaging - initial experience. Radiology 2005; 237: 67-74

101 Hundley WG, Morgan TM, Neagle CM et al. Magnetic resonance imaging determination of cardiac prognosis. Circulation 2002; 106 $2328-2333$

102 Duerinckx AJ, Atkinson D, Hurwitz R. Assessment of coronary artery patency after stent placement using magnetic resonance angiography. Journal Of Magnetic Resonance Imaging 1998; 8: 896-902

103 Hug J, Nagel E, Bornstedt A et al. Coronary arterial stents: Safety and artifacts during MR imaging. Radiology 2000; 216: 781 - 787

104 Maintz D, Botnar RM, Fischbach $R$ et al. Coronary magnetic resonance angiography for assessment of the stent lumen: a phantom study. J Cardiovasc Magn Reson 2002; 4: 359-367

105 Klem I, Heitner JF, Shah DJ et al. Improved detection of coronary artery disease by stress perfusion cardiovascular magnetic resonance with the use of delayed enhancement infarction imaging. J Am Coll Cardiol 2006; 47: $1630-1638$

106 Nagel E, Thouet T, Klein C et al. Noninvasive determination of coronary blood flow velocity with cardiovascular magnetic resonance in patients after stent deployment. Circulation 2003; 107: 1738-1743

107 Saito Y, Sakuma $H$, Shibata $M$ et al. Assessment of coronary flow velocity reserve using fast velocity-encoded cine MRI for noninvasive detection of restenosis after coronary stent implantation. J Cardiovasc Magn Reson 2001; 3: 209-214

108 Bernhardt P, Spiess J, Levenson B et al. Combined assessment of myocardial perfusion and late gadolinium enhancement in patients after percutaneous coronary intervention or bypass grafts: a multicenter study of an integrated cardiovascular magnetic resonance protocol. JACC Cardiovasc Imaging 2009; 2: $1292-1300$

109 Cury RC, Cattani CA, Gabure LA et al. Diagnostic performance of stress perfusion and delayed-enhancement MR imaging in patients with coronary artery disease. Radiology 2006; 240: 39-45

110 Doesch C, Seeger A, Hoevelborn T et al. Adenosine stress cardiac magnetic resonance imaging for the assessment of ischemic heart disease. Clin Res Cardiol 2008; 97: 905-912

111 Pilz G, Bernhardt $P$, Klos $M$ et al. Clinical implication of adenosinestress cardiac magnetic resonance imaging as potential gatekeeper prior to invasive examination in patients with AHA/ACC class II indication for coronary angiography. Clin Res Cardiol 2006; 95: 531 - 538

112 Steel $K$, Broderick R, Gandla V et al. Complementary prognostic values of stress myocardial perfusion and late gadolinium enhancement imaging by cardiac magnetic resonance in patients with known or suspected coronary artery disease. Circulation 2009; 120: 1390 1400 
113 Heilmaier C, Bruder O, Meier F et al. Dobutamine stress cardiovascular magnetic resonance imaging in patients after invasive coronary revascularization with stent placement. Acta Radiol 2009; 50: 1134 1141

114 Hundley WG, Hamilton CA, Thomas MS et al. Utility of fast cine magnetic resonance imaging and display for the detection of myocardial ischemia in patients not well suited for second harmonic stress echocardiography. Circulation 1999; 100: 1697-1702

115 Korosoglou G, Lossnitzer D, Schellberg $D$ et al. Strain-encoded cardiac MRI as an adjunct for dobutamine stress testing: incremental value to conventional wall motion analysis. Circ Cardiovasc Imaging 2009; 2: $132-140$

116 Kuijpers D, Ho KY, van Dijkman PR et al. Dobutamine cardiovascular magnetic resonance for the detection of myocardial ischemia with the use of myocardial tagging. Circulation 2003; 107: 1592-1597

117 Paetsch I, Jahnke C, Ferrari VA et al. Determination of interobserver variability for identifying inducible left ventricular wall motion abnormalities during dobutamine stress magnetic resonance imaging. Eur Heart J 2006; 27: 1459 - 1464

118 Wahl A, Paetsch I, Roethemeyer S et al. High-dose dobutamine-atropine stress cardiovascular MR imaging after coronary revascularization in patients with wall motion abnormalities at rest. Radiology 2004; 233: $210-216$

119 Dikkers $R$, Willems TP, de Jonge GJ et al. Accuracy of noninvasive coronary stenosis quantification of different commercially available dedicated software packages. J Comput Assist Tomogr 2009; 33: 505-512

120 Langerak SE, Vliegen HW, de Roos A et al. Detection of vein graft disease using high-resolution magnetic resonance angiography. Circulation 2002; 105: $328-333$

121 Langerak SE, Vliegen HW, Jukema JW et al. Value of magnetic resonance imaging for the noninvasive detection of stenosis in coronary artery bypass grafts and recipient coronary arteries. Circulation 2003; 107: 1502 - 1508

122 Langerak SE, Vliegen HW, Jukema JW et al. Vein graft function improvement after percutaneous intervention: evaluation with MR flow mapping. Radiology 2003; 228: 834-841

123 Salm LP, Bax JJ, Vliegen HW et al. Functional significance of stenoses in coronary artery bypass grafts. Evaluation by single-photon emission computed tomography perfusion imaging, cardiovascular magnetic resonance, and angiography. J Am Coll Cardiol 2004; 44: 1877-1882

124 Salm LP, Langerak SE, Vliegen HW et al. Blood flow in coronary artery bypass vein grafts: volume versus velocity at cardiovascular MR imaging. Radiology 2004; 232: 915 - 920

125 Salm LP, Vliegen HW, Langerak SE et al. Evaluation of saphenous vein coronary artery bypass graft flow by cardiovascular magnetic resonance. J Cardiovasc Magn Reson 2005; 7: 631 - 637

126 Stauder NI, Klumpp B, Stauder H et al. Assessment of coronary artery bypass grafts by magnetic resonance imaging. Br J Radiol 2007; 80: 975-983

127 Meyer TS, Martinoff S, Hadamitzky M et al. Improved noninvasive assessment of coronary artery bypass grafts with 64-slice computed tomographic angiography in an unselected patient population. J Am Coll Cardiol 2007; 49: 946 - 950

128 Onuma $Y$, Tanabe K, Chihara $R$ et al. Evaluation of coronary artery bypass grafts and native coronary arteries using 64-slice multidetector computed tomography. Am Heart J 2007; 154: 519-526

129 Weustink AC, Nieman K, Pugliese F et al. Diagnostic accuracy of computed tomography angiography in patients after bypass grafting: comparison with invasive coronary angiography. JACC Cardiovasc Imaging 2009; 2: 816-824

130 Ropers D, Pohle FK, Kuettner A et al. Diagnostic accuracy of noninvasive coronary angiography in patients after bypass surgery using 64slice spiral computed tomography with 330-ms gantry rotation. Circulation 2006; 114: 2334-2341; quiz

131 Nazeri I, Shahabi P, Tehrai $M$ et al. Assessment of patients after coronary artery bypass grafting using 64-slice computed tomography. Am J Cardiol 2009; 103: 667-673

132 Angelini P, Velasco JA, Flamm S. Coronary anomalies: incidence, pathophysiology, and clinical relevance. Circulation 2002; 105: 24492454

133 Vliegen HW, Doornbos J, de Roos A et al. Value of fast gradient echo magnetic resonance angiography as an adjunct to coronary arteriography in detecting and confirming the course of clinically significant coronary artery anomalies. Am J Cardiol 1997; 79: 773 - 776
134 Post JC, van Rossum AC, Bronzwaer JG et al. Magnetic resonance angiography of anomalous coronary arteries. A new gold standard for delineating the proximal course? Circulation 1995; 92: 3163-3171

135 Taylor AM, Thorne SA, Rubens MB et al. Coronary artery imaging in grown up congenital heart disease: complementary role of magnetic resonance and x-ray coronary angiography. Circulation 2000; 101: $1670-1678$

$136 \mathrm{Kacmaz}$ F, Ozbulbul NI, Alyan $\mathrm{O}$ et al. Imaging of coronary artery anomalies: the role of multidetector computed tomography. Coron Artery Dis 2008; 19: $203-209$

137 Shi H, Aschoff AJ, Brambs HJ et al. Multislice CT imaging of anomalous coronary arteries. Eur Radiol 2004; 14: 2172 - 2181

138 Duran C, Kantarci M, Durur Subasi I et al. Remarkable anatomic anomalies of coronary arteries and their clinical importance: a multidetector computed tomography angiographic study. J Comput Assist Tomogr 2006; 30: 939-948

139 Schmid M, Achenbach S, Ludwig J et al. Visualization of coronary artery anomalies by contrast-enhanced multi-detector row spiral computed tomography. Int J Cardiol 2006; 111: 430-435

$140 \mathrm{Kim} \mathrm{SY,} \mathrm{Seo} \mathrm{JB,} \mathrm{Do} \mathrm{KH} \mathrm{et} \mathrm{al.} \mathrm{Coronary} \mathrm{artery} \mathrm{anomalies:} \mathrm{classification}$ and ECG-gated multi-detector row CT findings with angiographic correlation. Radiographics 2006; 26: 317-333; discussion 33-34

141 Hachulla $A L$, Launay D, Gaxotte $V$ et al. Cardiac magnetic resonance imaging in systemic sclerosis: a cross-sectional observational study of 52 patients. Ann Rheum Dis 2009; 68: 1878-1884

142 Ichinose A, Otani $\mathrm{H}$, Oikawa $M$ et al. MRI of cardiac sarcoidosis: basal and subepicardial localization of myocardial lesions and their effect on left ventricular function. Am J Roentgenol 2008; 191: 862 - 869

143 Maceira AM, Prasad SK, Hawkins PN et al. Cardiovascular magnetic resonance and prognosis in cardiac amyloidosis. J Cardiovasc Magn Reson 2008; 10: 54

144 Mavrogeni S, Manoussakis MN, Karagiorga TC et al. Detection of coronary artery lesions and myocardial necrosis by magnetic resonance in systemic necrotizing vasculitides. Arthritis Rheum 2009; 61: 1121 1129

145 Patel MR, Cawley PJ, Heitner JF et al. Detection of myocardial damage in patients with sarcoidosis. Circulation 2009; 120: 1969-1977

146 Pepe A, Positano V, Santarelli MF et al. Multislice multiecho T2* cardiovascular magnetic resonance for detection of the heterogeneous distribution of myocardial iron overload. J Magn Reson Imaging 2006; 23: $662-668$

147 Vogelsberg H, Mahrholdt H, Deluigi CC et al. Cardiovascular magnetic resonance in clinically suspected cardiac amyloidosis: noninvasive imaging compared to endomyocardial biopsy. J Am Coll Cardiol 2008; 51: $1022-1030$

148 Thiele H, Nagel E, Paetsch I et al. Functional cardiac MR imaging with steady-state free precession (SSFP) significantly improves endocardial border delineation without contrast agents. J Magn Reson Imaging 2001; $14: 362-367$

149 Thiele H, Paetsch I, Schnackenburg B et al. Improved accuracy of quantitative assessment of left ventricular volume and ejection fraction by geometric models with steady-state free precession. J Cardiovasc Magn Reson 2002; 4: 327 - 339

150 Codreanu A, Djaballah W, Angioi M et al. Detection of myocarditis by contrast-enhanced MRI in patients presenting with acute coronary syndrome but no coronary stenosis. J Magn Reson Imaging 2007; 25: $957-964$

151 O'Hanlon R, Pennell DJ. Cardiovascular magnetic resonance in the evaluation of hypertrophic and infiltrative cardiomyopathies. Heart Fail Clin 2009; 5: 369-387, vi

152 Shehata ML, Turkbey EB, Vogel-Claussen J et al. Role of cardiac magnetic resonance imaging in assessment of nonischemic cardiomyopathies. Top Magn Reson Imaging 2008; 19: 43 - 57

$153 \mathrm{Wu}$ KC, Weiss RG, Thiemann DR et al. Late gadolinium enhancement by cardiovascular magnetic resonance heralds an adverse prognosis in nonischemic cardiomyopathy. J Am Coll Cardiol 2008; 51: 2414 2421

154 Henneman MM, Schuijf JD, Jukema JW et al. Assessment of global and regional left ventricular function and volumes with 64-slice MSCT: a comparison with 2D echocardiography. J Nucl Cardiol 2006; 13: 480-487

155 Yamamuro $M$, Tadamura $E$, Kubo $S$ et al. Cardiac functional analysis with multi-detector row CT and segmental reconstruction algorithm: comparison with echocardiography, SPECT, and MR imaging. Radiology 2005; 234: $381-390$ 
156 Grude M, Juergens KU, Wichter T et al. Evaluation of global left ventricular myocardial function with electrocardiogram-gated multidetector computed tomography: comparison with magnetic resonance imaging. Invest Radiol 2003; 38: 653-661

157 Raman SV, Cook SC, McCarthy B et al. Usefulness of multidetector row computed tomography to quantify right ventricular size and function in adults with either tetralogy of Fallot or transposition of the great arteries. Am J Cardiol 2005; 95: 683-686

158 Raman SV, Shah M, McCarthy B et al. Multi-detector row cardiac computed tomography accurately quantifies right and left ventricular size and function compared with cardiac magnetic resonance. Am Heart J 2006; 151: $736-744$

159 Hansen MW, Merchant N. MRI of hypertrophic cardiomyopathy: part 2, Differential diagnosis, risk stratification, and posttreatment MRI appearances. Am J Roentgenol 2007; 189: 1344-1352

160 Nazarian S, Lima JA. Cardiovascular magnetic resonance for risk stratification of arrhythmia in hypertrophic cardiomyopathy. J Am Coll Cardiol 2008; 51: $1375-1376$

161 Assomull RG, Prasad SK, Lyne J et al. Cardiovascular magnetic resonance, fibrosis, and prognosis in dilated cardiomyopathy. J Am Coll Cardiol 2006; 48: 1977 - 1985

162 Koikkalainen JR, Antila M, Lotjonen JM et al. Early familial dilated cardiomyopathy: identification with determination of disease state parameter from cine MR image data. Radiology 2008; 249: 88- 96

163 Shimizu I, Iguchi N, Watanabe H et al. Delayed enhancement cardiovascular magnetic resonance as a novel technique to predict cardiac events in dilated cardiomyopathy patients. Int J Cardiol 2010; 142: $224-229$

164 Giorgi B, Mollet NR, Dymarkowski S et al. Clinically suspected constrictive pericarditis: MR imaging assessment of ventricular septal motion and configuration in patients and healthy subjects. Radiology 2003; 228: $417-424$

165 Hancock EW. Differential diagnosis of restrictive cardiomyopathy and constrictive pericarditis. Heart 2001; 86: 343-349

166 Miller S, Riessen R. [MR imaging in cardiomyopathies]. Fortschr Röntgenstr 2005; 177: 1497-505

167 Moreo A, Ambrosio G, De Chiara B et al. Influence of myocardial fibrosis on left ventricular diastolic function: noninvasive assessment by cardiac magnetic resonance and echo. Circ Cardiovasc Imaging 2009; 2: $437-443$

168 Biagini E, Ragni L, Ferlito $M$ et al. Different types of cardiomyopathy associated with isolated ventricular noncompaction. Am J Cardio 2006; 98: $821-824$

169 Patlas M, Strohm O, Filipchuk N et al. Cardiac magnetic resonance imaging of noncompaction cardiomyopathy. Can J Cardiol 2008; 24: 798

170 Sen-Chowdhry S, Prasad SK, Syrris $P$ et al. Cardiovascular magnetic resonance in arrhythmogenic right ventricular cardiomyopathy revisited: comparison with task force criteria and genotype. J Am Coll Cardiol 2006; 48: $2132-2140$

171 White RD, Trohman RG, Flamm SD et al. Right ventricular arrhythmia in the absence of arrhythmogenic dysplasia: MR imaging of myocardial abnormalities. Radiology 1998; 207: 743 - 751

172 Crean A, Greenwood JP, Plein S. Contribution of noninvasive imaging to the diagnosis and follow-up of Takotsubo cardiomyopathy. JACC Cardiovasc Imaging 2009; 2: 519-521

173 Yilmaz A, Kindermann I, Kindermann $M$ et al. Comparative evaluation of left and right ventricular endomyocardial biopsy: differences in complication rate and diagnostic performance. Circulation 2010; 122: $900-909$

174 Gutberlet $M$, Spors B, Thoma $T$ et al. Suspected chronic myocarditis at cardiac MR: diagnostic accuracy and association with immunohistologically detected inflammation and viral persistence. Radiology 2008; 246: $401-409$

175 Mahrholdt H, Goedecke C, Wagner A et al. Cardiovascular magnetic resonance assessment of human myocarditis: a comparison to histology and molecular pathology. Circulation 2004; 109: 1250-1258

176 Mahrholdt H, Wagner A, Deluigi CC et al. Presentation, patterns of myocardial damage, and clinical course of viral myocarditis. Circulation 2006; 114: $1581-1590$

177 Friedrich MG, Sechtem U, Schulz-Menger J et al. Cardiovascular magnetic resonance in myocarditis: A JACC White Paper. J Am Coll Cardiol 2009; 53: 1475 - 1487

178 Lin $C H$, Chang WN, Chua S et al. Idiopathic hypereosinophilia syndrome with loeffler endocarditis, embolic cerebral infarction, and left hydranencephaly: a case report. Acta Neurol Taiwan 2009; 18: $207-212$

179 Germans T, van Rossum AC. The use of cardiac magnetic resonance imaging to determine the aetiology of left ventricular disease and cardiomyopathy. Heart 2008; 94: 510-518

180 Harris SR, Glockner J, Misselt AJ et al. Cardiac MR imaging of nonischemic cardiomyopathies. Magn Reson Imaging Clin N Am 2008; 16: $165-183$, vii

181 Duckett SG, Chiribiri A, Ginks MR et al. Cardiac MRI to investigate myocardial scar and coronary venous anatomy using a slow infusion of dimeglumine gadobenate in patients undergoing assessment for cardiac resynchronization therapy. J Magn Reson Imaging 2011; 33: 87 95

182 Chiribiri A, Kelle S, Köhler $U$ et al. Magnetic resonance cardiac vein imaging: relation to mitral valve annulus and left circumflex coronary artery. JACC Cardiovasc Imaging 2008; 1: 729-738

183 Jongbloed MR, Lamb HJ, Bax JJ et al. Noninvasive visualization of the cardiac venous system using multislice computed tomography. J Am Coll Cardiol 2005; 45: 749-753

$184 \mathrm{Kim}$ YH, Marom EM, Herndon JE et al. Pulmonary vein diameter, crosssectional area, and shape: CT analysis. Radiology 2005; 235: 43 -49; discussion 9-50

185 Van de Veire NR, Marsan NA, Schuijf JD et al. Noninvasive imaging of cardiac venous anatomy with 64-slice multi-slice computed tomography and noninvasive assessment of left ventricular dyssynchrony by 3-dimensional tissue synchronization imaging in patients with heart failure scheduled for cardiac resynchronization therapy. Am J Cardiol 2008; 101: 1023-1029

186 Choure AJ, Garcia MJ, Hesse B et al. In vivo analysis of the anatomical relationship of coronary sinus to mitral annulus and left circumflex coronary artery using cardiac multidetector computed tomography: implications for percutaneous coronary sinus mitral annuloplasty. J Am Coll Cardiol 2006; 48: 1938-1945

187 Tops LF, Van de Veire NR, Schuijf JD et al. Noninvasive evaluation of coronary sinus anatomy and its relation to the mitral valve annulus: implications for percutaneous mitral annuloplasty. Circulation 2007 115: $1426-1432$

188 Bleeker GB, Kaandorp TA, Lamb HJ et al. Effect of posterolateral scar tissue on clinical and echocardiographic improvement after cardiac resynchronization therapy. Circulation 2006; 113: 969-976

189 Delgado $V$, van Bommel RJ, Bertini $M$ et al. Relative merits of left ventricular dyssynchrony, left ventricular lead position, and myocardial scar to predict long-term survival of ischemic heart failure patients undergoing cardiac resynchronization therapy. Circulation 2011; 123: $70-78$

190 Marsan NA, Westenberg JJ, Ypenburg C et al. Magnetic resonance imaging and response to cardiac resynchronization therapy: relative merits of left ventricular dyssynchrony and scar tissue. Eur Heart J 2009; 30: $2360-2367$

191 White JA, Yee R, Yuan X et al. Delayed enhancement magnetic resonance imaging predicts response to cardiac resynchronization therapy in patients with intraventricular dyssynchrony. J Am Coll Cardiol 2006; 48: 1953 - 1960

192 Ypenburg C, Schalij MJ, Bleeker GB et al. Impact of viability and scar tissue on response to cardiac resynchronization therapy in ischaemic heart failure patients. Eur Heart J 2007; 28: 33-41

193 England B, Lee A, Tran T et al. Magnetic resonance criteria for future trials of cardiac resynchronization therapy. J Cardiovasc Magn Reson 2005; 7: $827-834$

194 Muellerleile K, Stork A, Bansmann $M$ et al. Detection of mechanical ventricular asynchrony by high temporal resolution cine MRI. Eur Radiol 2008; 18: 1329-1337

195 Zwanenburg JJ, Gotte MJ, Kuijer JP et al. Timing of cardiac contraction in humans mapped by high-temporal-resolution MRI tagging: early onset and late peak of shortening in lateral wall. Am J Physiol Heart Circ Physiol 2004; 286: H1872 - H1880

196 Truong QA, Singh JP, Cannon CP et al. Quantitative analysis of intraventricular dyssynchrony using wall thickness by multidetector computed tomography. JACC Cardiovasc Imaging 2008; 1: $772-781$

197 Choi EY, Choi BW, Kim SA et al. Patterns of late gadolinium enhancement are associated with ventricular stiffness in patients with advanced non-ischaemic dilated cardiomyopathy. Eur J Heart Fail 2009; 11: $573-580$ 
198 Maron MS, Appelbaum E, Harrigan CJ et al. Clinical profile and significance of delayed enhancement in hypertrophic cardiomyopathy. Circ Heart Fail 2008; 1: 184-191

199 Jain A, Tandri H, Calkins $H$ et al. Role of cardiovascular magnetic resonance imaging in arrhythmogenic right ventricular dysplasia. J Cardiovasc Magn Reson 2008; 10: 32

200 Tandri H, Saranathan M, Rodriguez ER et al. Noninvasive detection of myocardial fibrosis in arrhythmogenic right ventricular cardiomyopathy using delayed-enhancement magnetic resonance imaging. J Am Coll Cardiol 2005; 45: 98-103

201 Chyou JY, Biviano A, Magno P et al. Applications of computed tomography and magnetic resonance imaging in percutaneous ablation therapy for atrial fibrillation. J Interv Card Electrophysiol 2009; 26: 47 57

202 Hamdan A, Charalampos K, Roettgen $R$ et al. Magnetic resonance imaging versus computed tomography for characterization of pulmonary vein morphology before radiofrequency catheter ablation of atrial fibrillation. Am J Cardiol 2009; 104: 1540-1546

203 Mansour M, Holmvang G, Sosnovik D et al. Assessment of pulmonary vein anatomic variability by magnetic resonance imaging: implications for catheter ablation techniques for atrial fibrillation. J Cardiovasc Electrophysiol 2004; 15: 387-393

204 Jongbloed MR, Dirksen MS, Bax JJ et al. Atrial fibrillation: multi-detector row $\mathrm{CT}$ of pulmonary vein anatomy prior to radiofrequency catheter ablation - initial experience. Radiology 2005; 234: 702 - 709

205 Martinek M, Nesser HJ, Aichinger J et al. Accuracy of integration of multislice computed tomography imaging into three-dimensional electroanatomic mapping for real-time guided radiofrequency ablation of left atrial fibrillation-influence of heart rhythm and radiofrequency lesions. J Interv Card Electrophysiol 2006; 17: 85-92

206 Martinek M, Nesser HJ, Aichinger J et al. Impact of integration of multislice computed tomography imaging into three-dimensional electroanatomic mapping on clinical outcomes, safety, and efficacy using radiofrequency ablation for atrial fibrillation. Pacing Clin Electrophysiol 2007; 30: 1215-1223

207 Jongbloed MR, Bax JJ, Lamb HJ et al. Multislice computed tomography versus intracardiac echocardiography to evaluate the pulmonary veins before radiofrequency catheter ablation of atrial fibrillation: a head-to-head comparison. J Am Coll Cardiol 2005; 45: 343 - 350

208 Kistler PM, Rajappan K, Jahngir M et al. The impact of CT image integration into an electroanatomic mapping system on clinical outcomes of catheter ablation of atrial fibrillation. J Cardiovasc Electrophysiol 2006; 17: $1093-1101$

209 Dill T, Neumann T, Ekinci $O$ et al. Pulmonary vein diameter reduction after radiofrequency catheter ablation for paroxysmal atrial fibrillation evaluated by contrast-enhanced three-dimensional magnetic resonance imaging. Circulation 2003; 107: 845-850

210 Kluge A, Dill T, Ekinci $O$ et al. Decreased pulmonary perfusion in pulmonary vein stenosis after radiofrequency ablation: assessment with dynamic magnetic resonance perfusion imaging. Chest 2004; 126: $428-437$

211 Neumann T, Kuniss M, Conradi $G$ et al. Pulmonary vein stenting for the treatment of acquired severe pulmonary vein stenosis after pulmonary vein isolation: clinical implications after long-term follow-up of 4 years. J Cardiovasc Electrophysiol 2009; 20: 251-257

212 Packer DL, Keelan P, Munger TM et al. Clinical presentation, investigation, and management of pulmonary vein stenosis complicating ablation for atrial fibrillation. Circulation 2005; 111: 546-554

213 Peters DC, Wylie JV, Hauser TH et al. Recurrence of atrial fibrillation correlates with the extent of post-procedural late gadolinium enhancement: a pilot study. JACC Cardiovasc Imaging 2009; 2: 308-316

214 Barrett CD, Di Biase L, Natale A. How to identify and treat patient with pulmonary vein stenosis post atrial fibrillation ablation. Curr Opin Cardiol 2009; 24: 42 - 49

215 Burgstahler C, Trabold T, Kuettner A et al. Visualization of pulmonary vein stenosis after radio frequency ablation using multi-slice computed tomography: initial clinical experience in 33 patients. Int J Cardiol 2005; 102: 287-291

216 Malyar NM, Schlosser T, Barkhausen J et al. Assessment of aortic valve area in aortic stenosis using cardiac magnetic resonance tomography: comparison with echocardiography. Cardiology 2008; 109 : $126-134$

217 O'Brien KR, Gabriel RS, Greiser A et al. Aortic valve stenotic area calculation from phase contrast cardiovascular magnetic resonance: the importance of short echo time. J Cardiovasc Magn Reson 2009; 11: 49
218 Alkadhi H, Wildermuth S, Plass A et al. Aortic stenosis: comparative evaluation of 16-detector row CT and echocardiography. Radiology 2006; 240: 47-55

219 Halpern EJ, Mallya R, Sewell M et al. Differences in aortic valve area measured with CT planimetry and echocardiography (continuity equation) are related to divergent estimates of left ventricular outflow tract area. Am J Roentgenol 2009; 192: 1668 - 1673

220 LaBounty TM, Sundaram B, Agarwal P et al. Aortic valve area on 64MDCT correlates with transesophageal echocardiography in aortic stenosis. Am J Roentgenol 2008; 191: 1652 - 1658

221 Pouleur AC, le Polain de Waroux JB, Pasquet A et al. Aortic valve area assessment: multidetector CT compared with cine MR imaging and transthoracic and transesophageal echocardiography. Radiology 2007; 244: $745-754$

222 Gelfand EV, Hughes S, Hauser TH et al. Severity of mitral and aortic regurgitation as assessed by cardiovascular magnetic resonance: optimizing correlation with Doppler echocardiography. J Cardiovasc Magn Reson 2006; 8: 503-507

223 Kozerke S, Schwitter J, Pedersen EM et al. Aortic and mitral regurgitation: quantification using moving slice velocity mapping. J Magn Reson Imaging $2001 ; 14: 106-112$

224 Pouleur AC, le Polain de Waroux JB, Pasquet A et al. Planimetric and continuity equation assessment of aortic valve area: Head to head comparison between cardiac magnetic resonance and echocardiography. J Magn Reson Imaging 2007; 26: 1436-1443

225 Tanaka K, Makaryus AN, Wolff SD. Correlation of aortic valve area obtained by the velocity-encoded phase contrast continuity method to direct planimetry using cardiovascular magnetic resonance. J Cardiovasc Magn Reson 2007; 9: 799-805

226 Lin SJ, Brown PA, Watkins MP et al. Quantification of stenotic mitral valve area with magnetic resonance imaging and comparison with Doppler ultrasound. J Am Coll Cardiol 2004; 44: 133-137

227 Kon MW, Myerson SG, Moat NE et al. Quantification of regurgitant fraction in mitral regurgitation by cardiovascular magnetic resonance: comparison of techniques. J Heart Valve Dis 2004; 13: 600-607

228 Kilner PJ, Sievers B, Meyer GP et al. Double-chambered right ventricle or sub-infundibular stenosis assessed by cardiovascular magnetic resonance. J Cardiovasc Magn Reson 2002; 4: 373-379

229 Kivelitz DE, Dohmen PM, Lembcke A et al. Visualization of the pulmonary valve using cine MR imaging. Acta Radiol 2003; 44: 172-176

230 Rebergen SA, Chin JG, Ottenkamp J et al. Pulmonary regurgitation in the late postoperative follow-up of tetralogy of Fallot. Volumetric quantitation by nuclear magnetic resonance velocity mapping. Circulation 1993; 88: 2257-2266

231 Therrien J, Provost $Y$, Merchant $N$ et al. Optimal timing for pulmonary valve replacement in adults after tetralogy of Fallot repair. Am J Cardiol 2005; 95: 779-782

232 Stollberger C, Kopsa W, Finsterer J. Non-compaction of the right atrium and left ventricle in Ebstein's malformation. J Heart Valve Dis 2006; 15: 719-720

233 Reynier C, Garcier J, Legault B et al. [Cross-sectional imaging of post endocarditis paravalvular myocardial abscesses of native mitral valves: 4 cases]. J Radiol 2001; 82: 665-669

234 LaBounty TM, Agarwal PP, Chughtai A et al. Hemodynamic and functional assessment of mechanical aortic valves using combined echocardiography and multidetector computed tomography. J Cardiovasc Comput Tomogr 2009; 3: 161 - 167

235 Schultz CJ, Weustink A, Piazza N et al. Geometry and degree of apposition of the CoreValve ReValving system with multislice computed tomography after implantation in patients with aortic stenosis. J Am Coll Cardiol 2009; 54: $911-918$

236 Harris KM, Ang E, Lesser JR et al. Cardiac magnetic resonance imaging for detection of an abscess associated with prosthetic valve endocarditis: a case report. Heart Surg Forum 2007; 10: E186-E187

237 Meijboom WB, Mollet NR, Van Mieghem CA et al. Pre-operative computed tomography coronary angiography to detect significant coronary artery disease in patients referred for cardiac valve surgery. J Am Coll Cardiol 2006; 48: 1658 -1665

238 Tops LF, Delgado V, van der Kley F et al. Percutaneous aortic valve therapy: clinical experience and the role of multi-modality imaging. Heart 2009; 95: $1538-1546$

239 Wood DA, Tops LF, Mayo JR et al. Role of multislice computed tomography in transcatheter aortic valve replacement. Am J Cardiol 2009; 103: $1295-1301$ 
240 Tops LF, Wood DA, Delgado V et al. Noninvasive evaluation of the aortic root with multislice computed tomography implications for transcatheter aortic valve replacement. JACC Cardiovasc Imaging 2008; 1 : $321-330$

241 Kahlert $P$, Plicht B, Janosi RA et al. The role of imaging in percutaneous mitral valve repair. Herz 2009; 34: 458-467

242 Delgado V, Tops LF, Schuijf JD et al. Assessment of mitral valve anatomy and geometry with multislice computed tomography. JACC Cardiovasc Imaging 2009; 2: 556-565

243 Rienmuller R, Groll R, Lipton MJ. CT and MR imaging of pericardial disease. Radiol Clin North Am 2004; 42: 587-601, vi

244 Wang ZJ, Reddy GP, Gotway MB et al. CT and MR imaging of pericardial disease. Radiographics 2003; 23: S167-S180

245 Rifkin RD, Mernoff DB. Noninvasive evaluation of pericardial effusion composition by computed tomography. Am Heart J 2005; 149: $1120-1127$

246 Francone $M$, Dymarkowski S, Kalantzi $M$ et al. Magnetic resonance imaging in the evaluation of the pericardium. A pictorial essay. Radiol Med 2005; 109: 64-74; quiz 5-6

247 Misselt AJ, Harris SR, Glockner J et al. MR imaging of the pericardium. Magn Reson Imaging Clin N Am 2008; 16: 185 - 199, vii

248 Smith WH, Beacock DJ, Goddard AJ et al. Magnetic resonance evaluation of the pericardium. Br J Radiol 2001; 74: 384-392

249 Troughton RW, Asher CR, Klein AL. Pericarditis. Lancet 2004; 363: $717-727$

250 Bauner K, Horng A, Schmitz C et al. New observations from MR velocity-encoded flow measurements concerning diastolic function in constrictive pericarditis. Eur Radiol 2010; 20: 1831 - 1840

251 Francone M, Dymarkowski S, Kalantzi M et al. Assessment of ventricular coupling with real-time cine MRI and its value to differentiate constrictive pericarditis from restrictive cardiomyopathy. Eur Radiol 2006; 16: $944-951$

252 Myers RB, Spodick DH. Constrictive pericarditis: clinical and pathophysiologic characteristics. Am Heart J 1999; 138: 219-232

253 Nishimura RA. Constrictive pericarditis in the modern era: a diagnostic dilemma. Heart 2001; 86: 619-623

254 Suh SY, Rha SW, Kim JW et al. The usefulness of three-dimensional multidetector computed tomography to delineate pericardial calcification in constrictive pericarditis. Int J Cardiol 2006; 113: 414-416

255 Hoffmann $M H$, Shi $H$, Lieberknecht $M$ et al. Images in cardiovascular medicine. Sixteen-slice computed tomography and magnetic resonance imaging of calcified pericardium. Circulation 2003; 108: e48 e49

256 Khan NU, Yonan N. Does preoperative computed tomography reduce the risks associated with re-do cardiac surgery? Interact Cardiovasc Thorac Surg 2009; 9: 119-123

257 Schwefer M, Aschenbach $R$, Heidemann J et al. Constrictive pericarditis, still a diagnostic challenge: comprehensive review of clinical management. Eur J Cardiothorac Surg 2009; 36: 502 - 510

258 Chiles C, Woodard PK, Gutierrez FR et al. Metastatic involvement of the heart and pericardium: CT and MR imaging. Radiographics 2001; 21: 439- 449

259 Grebenc ML, Rosado de Christenson ML, Burke AP et al. Primary cardiac and pericardial neoplasms: radiologic-pathologic correlation. Radiographics 2000; 20: 1073-1103

260 Syed IS, Feng D, Harris SR et al. MR imaging of cardiac masses. Magn Reson Imaging Clin N Am 2008; 16: 137 - 164, vii

$261 \mathrm{Kim}$ JS, Kim HH, Yoon Y. Imaging of pericardial diseases. Clin Radiol 2007; 62: 626-631

262 Ling LH, Oh JK, Schaff HV et al. Constrictive pericarditis in the modern era: evolving clinical spectrum and impact on outcome after pericardiectomy. Circulation 1999; 100: 1380 - 1386

263 Sengupta PP, Eleid MF, Khandheria BK. Constrictive pericarditis. Circ J 2008; 72: 1555 - 1562

264 Spottiswoode B, Russell JB, Moosa S et al. Abnormal diastolic and systolic septal motion following pericardiectomy demonstrated by cine DENSE MRI. Cardiovasc J Afr 2008; 19: 208-209

265 Agelopoulou P, Kapatais A, Varounis $C$ et al. Hepatocellular carcinoma with invasion into the right atrium. Report of two cases and review of the literature. Hepatogastroenterology 2007; 54: 2106-2108

266 Hoffmann U, Globits S, Schima $W$ et al. Usefulness of magnetic resonance imaging of cardiac and paracardiac masses. Am J Cardiol 2003; 92: $890-895$
267 Juan O, Esteban E, Sotillo J et al. Atrial flutter and myocardial infarction-like ECG changes as manifestations of left ventricle involvement from lung carcinoma. Clin Transl Oncol 2008; 10: 125-127

268 Schnarkowski P, Wallner B, von Gumppenberg R et al. [Magnetic resonance tomography and magnetic resonance angiography of an infiltration of the left and right atria by a liver metastasis]. Röntgenpraxis 1992; 45: 98 - 99

269 Vanheste $R$, Vanhoenacker P, D'Haenens P. Primary cardiac lymphoma. JBR-BTR 2007; 90: 109-111

270 van Beek EJ, Stolpen AH, Khanna G et al. CT and MRI of pericardial and cardiac neoplastic disease. Cancer Imaging 2007; 7: 19-26

271 Kim EY, Choe YH, Sung K et al. Multidetector CT and MR imaging of cardiac tumors. Korean J Radiol 2009; 10: 164-175

272 Krombach GA, Spuentrup E, Buecker A et al. [Heart tumors: magnetic resonance imaging and multislice spiral CT]. Fortschr Röntgenstr 2005; 177: 1205 - 1218

273 Kraemer N, Balzer JC, Schoth F et al. [Atrial tumors in cardiac MRI] Fortschr Röntgenstr 2009; 181: 1038-1049

274 Mohrs OK, Nowak B, Petersen SE et al. Thrombus detection in the left atrial appendage using contrast-enhanced MRI: a pilot study. Am J Roentgenol 2006; 186: 198-205

275 Hur J, Kim YJ, Lee HJ et al. Left atrial appendage thrombi in stroke patients: detection with two-phase cardiac CT angiography versus transesophageal echocardiography. Radiology 2009; 251: 683-690

276 Hur J, Kim YJ, Lee HJ et al. Cardiac computed tomographic angiography for detection of cardiac sources of embolism in stroke patients. Stroke 2009; 40: 2073 - 2078

277 Hur J, Kim YJ, Nam JE et al. Thrombus in the left atrial appendage in stroke patients: detection with cardiac CT angiography - a preliminary report. Radiology 2008; 249: $81-87$

278 Martinez MW, Kirsch J, Williamson EE et al. Utility of nongated multidetector computed tomography for detection of left atrial thrombus in patients undergoing catheter ablation of atrial fibrillation. JACC Cardiovasc Imaging 2009; 2: 69-76

279 Feuchtner GM, Dichtl W, Bonatti JO et al. Diagnostic accuracy of cardiac 64-slice computed tomography in detecting atrial thrombi. Comparative study with transesophageal echocardiography and cardiac surgery. Invest Radiol 2008; 43: $794-801$

280 Barkhausen J, Hunold P, Eggebrecht $H$ et al. Detection and characterization of intracardiac thrombi on MR imaging. Am J Roentgenol 2002: 179: 1539 - 1544

281 Bruder O, Waltering KU, Hunold P et al. [Detection and characterization of left ventricular thrombi by MRI compared to transthoracic echocardiography]. Fortschr Röntgenstr 2005; 177: 344-349

282 Paydarfar D, Krieger D, Dib $N$ et al. In vivo magnetic resonance imaging and surgical histopathology of intracardiac masses: distinct features of subacute thrombi. Cardiology 2001; 95: 40-47

283 Weinsaft JW, Kim HW, Shah DJ et al. Detection of left ventricular thrombus by delayed-enhancement cardiovascular magnetic resonance prevalence and markers in patients with systolic dysfunction. J Am Coll Cardiol 2008; 52: $148-157$

284 Weinsaft JW, Kim RJ, Ross $M$ et al. Contrast-enhanced anatomic imaging as compared to contrast-enhanced tissue characterization for detection of left ventricular thrombus. JACC Cardiovasc Imaging 2009; 2: $969-979$

285 Araoz PA, Eklund HE, Welch TJ et al. CT and MR imaging of primary cardiac malignancies. Radiographics 1999; 19: 1421 - 1434

286 Kaminaga T, Takeshita T, Kimura I. Role of magnetic resonance imaging for evaluation of tumors in the cardiac region. Eur Radiol 2003; 13: L1 - L10

287 O'Donnell DH, Abbara S, Chaithiraphan V et al. Cardiac tumors: optimal cardiac MR sequences and spectrum of imaging appearances. Am J Roentgenol 2009; 193: 377-387

288 Strotmann J. [Cardiac tumors - clinical symptoms, diagnostic approaches, and therapeutic aspects]. Med Klin 2008; 103: 175 - 180

289 Henrikson CA, Leng CT, Yuh DD et al. Computed tomography to assess possible cardiac lead perforation. Pacing Clin Electrophysiol 2006; 29: $509-511$

290 Hirschl DA, Jain VR, Spindola-Franco H et al. Prevalence and characterization of asymptomatic pacemaker and ICD lead perforation on CT. Pacing Clin Electrophysiol 2007; 30: 28-32

291 Burgstahler C, Wohrle J, Kochs $M$ et al. Magnetic resonance imaging to assess acute changes in atrial and ventricular parameters after transcatheter closure of atrial septal defects. J Magn Reson Imaging 2007; 25: $1136-1140$ 
292 Mohrs OK, Petersen SE, Erkapic D et al. Diagnosis of patent foramen ovale using contrast-enhanced dynamic MRI: a pilot study. Am J Roentgenol 2005; 184: 234-240

293 Mohrs OK, Petersen SE, Erkapic D et al. Dynamic contrast-enhanced MRI before and after transcatheter occlusion of patent foramen ovale. Am J Roentgenol 2007; 188: 844-849

294 Nusser T, Hoher M, Merkle $N$ et al. Cardiac magnetic resonance imaging and transesophageal echocardiography in patients with transcatheter closure of patent foramen ovale. J Am Coll Cardiol 2006; 48: $322-329$

295 Weber C, Weber M, Ekinci $O$ et al. Atrial septal defects type II: noninvasive evaluation of patients before implantation of an Amplatzer Septal Occluder and on follow-up by magnetic resonance imaging compared with TEE and invasive measurement. Eur Radiol 2008; 18: $2406-2413$

296 Sarikouch S, Peters B, Gutberlet M et al. Sex-specific pediatric percentiles for ventricular size and mass as reference values for cardiac MRI: assessment by steady-state free-precession and phase-contrast MRI flow. Circ Cardiovasc Imaging 2010; 3: 65-76

297 Vashist S, Singh GK. Acute myocarditis in children: current concepts and management. Curr Treat Options Cardiovasc Med 2009; 11: $383-391$

298 Siegel MJ. Multiplanar and three-dimensional multi-detector row CT of thoracic vessels and airways in the pediatric population. Radiology 2003; 229: 641 - 650

299 Gilkeson RC, Markowitz AH, Ciancibello L. Multisection CT evaluation of the reoperative cardiac surgery patient. Radiographics 2003; 23: $\mathrm{S} 3-\mathrm{S} 17$

300 Eichhorn J, Fink C, Delorme S et al. Rings, slings and other vascular abnormalities. Ultrafast computed tomography and magnetic resonance angiography in pediatric cardiology. Z Kardiol 2004; 93: 201 208

301 Eichhorn JG, Fink C, Long F et al. [Multidetector CT for the diagnosis of congenital vascular anomalies and associated complications in newborns and infants]. Fortschr Röntgenstr 2005; 177: 1366-1372

302 Eichhorn JG, Ley S. [Congenital abnormalities of the aorta in children and adolescents]. Radiologe 2007; 47: 974-981

303 Eichhorn JG, Long FR, Hill SL et al. Assessment of in-stent stenosis in small children with congenital heart disease using multi-detector computed tomography: a validation study. Catheter Cardiovasc Interv 2006; 68: $11-20$

304 Gutberlet M, Abdul-Khaliq H, Grothoff $M$ et al. [Evaluation of left ventricular volumes in patients with congenital heart disease and abnormal left ventricular geometry. Comparison of MRI and transthoracic 3-dimensional echocardiography]. Fortschr Röntgenstr 2003; 175: $942-951$

305 Sarikouch S, Koerperich H, Boethig D et al. Reference values for atrial size and function in children and young adults by cardiac MR: a study of the German competence network congenital heart defects. J Magn Reson Imaging 2011; 33: 1028 -1039

306 Cohen MS, Anderson RH, Cohen MI et al. Controversies, genetics, diagnostic assessment, and outcomes relating to the heterotaxy syndrome. Cardiol Young 2007; 17: 29-43

307 Geva T, Vick GW3rd, Wendt RE et al. Role of spin echo and cine magnetic resonance imaging in presurgical planning of heterotaxy syndrome. Comparison with echocardiography and catheterization. Circulation 1994; 90: $348-356$

308 Hong YK, Park YW, Ryu SJ et al. Efficacy of MRI in complicated congenital heart disease with visceral heterotaxy syndrome. J Comput Assist Tomogr 2000; 24: 671 -682

309 Lee EY, Zurakowski D, Waltz DA et al. MDCT evaluation of the prevalence of tracheomalacia in children with mediastinal aortic vascular anomalies. J Thorac Imaging 2008; 23: 258-265

310 Lee EY, Siegel MJ, Hildebolt CF et al. MDCT evaluation of thoracic aortic anomalies in pediatric patients and young adults: comparison of axial, multiplanar, and 3D images. Am J Roentgenol 2004; 182: 777 - 784

311 Ou P, Marini D, Celermajer DS et al. Non-invasive assessment of congenital pulmonary vein stenosis in children using cardiac-non-gated CT with 64-slice technology. Eur J Radiol 2009; 70: 595-599

312 Ou P, Celermajer DS, Calcagni G et al. Three-dimensional CT scanning: a new diagnostic modality in congenital heart disease. Heart 2007; 93: $908-913$

313 Oh KH, Choo KS, Lim SJ et al. Multidetector CT evaluation of total anomalous pulmonary venous connections: comparison with echocardiography. Pediatr Radiol 2009; 39: 950-954
314 Gilkeson RC, Ciancibello L, Zahka K. Pictorial essay. Multidetector CT evaluation of congenital heart disease in pediatric and adult patients. Am J Roentgenol 2003; 180: 973 - 980

315 Selby JB, Poghosyan T, Wharton M. Asymptomatic partial anomalous pulmonary venous return masquerading as pulmonary vein occlusion following radiofrequency ablation. Int J Cardiovasc Imaging 2006; 22: $719-722$

316 Wang XM, Wu LB, Sun C et al. Clinical application of 64-slice spiral CT in the diagnosis of the Tetralogy of Fallot. Eur J Radiol 2007; 64: 296 301

317 Beerbaum $P$, Korperich $H$, Barth $P$ et al. Noninvasive quantification of left-to-right shunt in pediatric patients: phase-contrast cine magnetic resonance imaging compared with invasive oximetry. Circulation 2001; 103: $2476-2482$

318 Beerbaum $P$, Korperich $H$, Esdorn $H$ et al. Atrial septal defects in pediatric patients: noninvasive sizing with cardiovascular MR imaging. Radiology 2003; 228: 361 - 369

319 Durongpisitkul K, Tang NL, Soongswang J et al. Predictors of successful transcatheter closure of atrial septal defect by cardiac magnetic resonance imaging. Pediatr Cardiol 2004; 25: 124-130

320 Piaw CS, Kiam OT, Rapaee A et al. Use of non-invasive phase contrast magnetic resonance imaging for estimation of atrial septal defect size and morphology: a comparison with transesophageal echo. Cardiovasc Intervent Radiol 2006; 29: 230-234

321 Thomson LE, Crowley AL, Heitner JF et al. Direct en face imaging of secundum atrial septal defects by velocity-encoded cardiovascular magnetic resonance in patients evaluated for possible transcatheter closure. Circ Cardiovasc Imaging 2008; $1: 31$ - 40

322 Valente AM, Sena L, Powell AJ et al. Cardiac magnetic resonance imaging evaluation of sinus venosus defects: comparison to surgical findings. Pediatr Cardiol 2007; 28: 51 - 56

323 Rajiah P, Kanne JP. Computed tomography of septal defects. J Cardiovasc Comput Tomogr 2010; 4: 231 - 245

324 Beerbaum P, Parish V, Bell A et al. Atypical atrial septal defects in children: noninvasive evaluation by cardiac MRI. Pediatr Radiol 2008; 38 : $1188-1194$

325 Grosse-Wortmann L, Al-Otay A, Goo HW et al. Anatomical and functional evaluation of pulmonary veins in children by magnetic resonance imaging. J Am Coll Cardiol 2007; 49: 993-1002

326 Riesenkampff EM, Schmitt B, Schnackenburg B et al. Partial anomalous pulmonary venous drainage in young pediatric patients: the role of magnetic resonance imaging. Pediatr Cardiol 2009; 30: 458-464

327 Bass JE, Redwine MD, Kramer LA et al. Spectrum of congenital anomalies of the inferior vena cava: cross-sectional imaging findings. Radiographics 2000; 20: 639-652

328 Greil GF, Powell AJ, Gildein HP et al. Gadolinium-enhanced three-dimensional magnetic resonance angiography of pulmonary and systemic venous anomalies. J Am Coll Cardiol 2002; 39: 335 - 341

329 Kersting-Sommerhoff BA, Diethelm L, Teitel DF et al. Magnetic resonance imaging of congenital heart disease: sensitivity and specificity using receiver operating characteristic curve analysis. Am Heart J 1989; 118: 155 - 161

330 Sorensen TS, Korperich H, Greil GF et al. Operator-independent isotropic three-dimensional magnetic resonance imaging for morphology in congenital heart disease: a validation study. Circulation 2004; 110: 163 - 169

331 Brown ML, Dearani JA, Danielson GK et al. The outcomes of operations for 539 patients with Ebstein anomaly. J Thorac Cardiovasc Surg 2008; 135: $1120-36$

332 Malhotra SP, Petrossian E, Reddy VM et al. Selective right ventricular unloading and novel technical concepts in Ebstein's anomaly. Ann Thorac Surg 2009; 88: 1975 - 1981

333 Gutberlet M, Oellinger H, Ewert P et al. [Pre- and postoperative evaluation of ventricular function, muscle mass and valve morphology by magnetic resonance tomography in Ebstein's anomaly]. Fortschr Röntgenstr 2000; 172: 436-442

334 Bell A, Beerbaum P, Greil G et al. Noninvasive assessment of pulmonary artery flow and resistance by cardiac magnetic resonance in congenital heart diseases with unrestricted left-to-right shunt. JACC Cardiovasc Imaging 2009; 2: 1285 - 1291

335 Grosse-Wortmann L, Yun TJ, Al-Radi 0 et al. Borderline hypoplasia of the left ventricle in neonates: insights for decision-making from functional assessment with magnetic resonance imaging. J Thorac Cardiovasc Surg 2008; 136: 1429-1436 
336 Shuhaiber JH, Ho SY, Rigby M et al. Current options and outcomes for the management of atrioventricular septal defect. Eur J Cardiothorac Surg 2009; 35: $891-900$

337 Marijon E, Ou P, Fermont L et al. Diagnosis and outcome in congenital ventricular diverticulum and aneurysm. J Thorac Cardiovasc Surg 2006; 131: $433-437$

338 McMahon CJ, Moniotte S, Powell AJ et al. Usefulness of magnetic resonance imaging evaluation of congenital left ventricular aneurysms. Am J Cardiol 2007; 100: 310-315

339 Ohlow MA. Congenital left ventricular aneurysms and diverticula: definition, pathophysiology, clinical relevance and treatment. Cardiology 2006; 106: $63-72$

340 Hoppe UC, Dederichs B, Deutsch HJ et al. Congenital heart disease in adults and adolescents: comparative value of transthoracic and transesophageal echocardiography and MR imaging. Radiology 1996; 199: 669-677

341 Kellenberger CJ, Yoo SJ, Buchel ER. Cardiovascular MR imaging in neonates and infants with congenital heart disease. Radiographics 2007; 27: $5-18$

342 Kersting-Sommerhoff BA, Diethelm L, Stanger $P$ et al. Evaluation of complex congenital ventricular anomalies with magnetic resonance imaging. Am Heart J 1990; 120: $133-142$

343 Kersting-Sommerhoff BA, Seelos KC, Hardy C et al. Evaluation of surgical procedures for cyanotic congenital heart disease by using MR imaging. Am J Roentgenol 1990; 155: 259 - 266

344 Kilner PJ, Geva T, Kaemmerer H et al. Recommendations for cardiovascular magnetic resonance in adults with congenital heart disease from the respective working groups of the European Society of Cardiology. Eur Heart J 2010; 31: 794 - 805

345 Rutledge JM, Nihill MR, Fraser CD et al. Outcome of 121 patients with congenitally corrected transposition of the great arteries. Pediatr Cardiol 2002; 23: $137-145$

346 Salehian 0, Schwerzmann M, Merchant N et al. Assessment of systemic right ventricular function in patients with transposition of the great arteries using the myocardial performance index: comparison with cardiac magnetic resonance imaging. Circulation 2004; 110: 3229 3233

347 Samyn MM. A review of the complementary information available with cardiac magnetic resonance imaging and multi-slice computed tomography (CT) during the study of congenital heart disease. Int Cardiovasc Imaging 2004; 20: $569-578$

348 Sarikouch S, Schaeffler $R$, Korperich $H$ et al. Cardiovascular magnetic resonance imaging for intensive care infants: safe and effective? Pediatr Cardiol 2009; 30: 146-152

349 Wood JC. Anatomical assessment of congenital heart disease. J Cardiovasc Magn Reson 2006; 8: 595-606

350 Chaturvedi RR, Redington AN. Pulmonary regurgitation in congenital heart disease. Heart 2007; 93: 880-889

351 Geva T. Indications and timing of pulmonary valve replacement after tetralogy of Fallot repair. Semin Thorac Cardiovasc Surg Pediatr Card Surg Annu 2006, 11 - 22

352 Harrild DM, Berul CI, Cecchin F et al. Pulmonary valve replacement in tetralogy of Fallot: impact on survival and ventricular tachycardia. Circulation 2009; 119: 445 - 451

353 Henkens IR, van Straten A, Schalij MJ et al. Predicting outcome of pulmonary valve replacement in adult tetralogy of Fallot patients. Ann Thorac Surg 2007; 83: 907 - 911

354 Oosterhof T, van Straten A, Vliegen HW et al. Preoperative thresholds for pulmonary valve replacement in patients with corrected tetralogy of Fallot using cardiovascular magnetic resonance. Circulation 2007; 116: 545 - 551

355 Vliegen HW, van Straten A, de Roos A et al. Magnetic resonance imaging to assess the hemodynamic effects of pulmonary valve replacement in adults late after repair of tetralogy of fallot. Circulation 2002; 106: $1703-1707$

356 Dincer TC, Basarici I, Calisir C et al. Ruptured aneurysm of noncoronary sinus of Valsalva: demonstration with magnetic resonance imaging. Acta Radiol 2008; 49: 889-892

357 Feldman DN, Roman MJ. Aneurysms of the sinuses of Valsalva. Cardiology 2006; 106: $73-81$

358 Karaaslan T, Gudinchet F, Payot $M$ et al. Congenital aneurysm of sinus of valsalva ruptured into right ventricle diagnosed by magnetic resonance imaging. Pediatr Cardiol 1999; 20: 212-214

359 Ozkara A, Cetin G, Mert $M$ et al. Sinus of Valsalva aneurysm: surgical approaches to complicated cases. ANZ J Surg 2005; 75: 51 - 54
360 Bricker AO, Avutu B, Mohammed TL et al. Valsalva sinus aneurysms: findings at CT and MR imaging. Radiographics 2010; 30: 99-110

361 Crean A. Cardiovascular MR and CT in congenital heart disease. Heart 2007; 93: 1637 - 1647

362 Dillman JR, Yarram SG, D'Amico AR et al. Interrupted aortic arch: spectrum of MRI findings. Am J Roentgenol 2008; 190: 1467-1474

363 Eichhorn JG, Fink C, Delorme S et al. Magnetic resonance blood flow measurements in the follow-up of pediatric patients with aortic coarctation - a re-evaluation. Int J Cardiol 2006; 113: 291 -298

364 Geva T, Greil GF, Marshall AC et al. Gadolinium-enhanced 3-dimensional magnetic resonance angiography of pulmonary blood supply in patients with complex pulmonary stenosis or atresia: comparison with x-ray angiography. Circulation 2002; 106: 473-478

365 Grosse-Wortmann L, Al-Otay A, Yoo SJ. Aortopulmonary collaterals after bidirectional cavopulmonary connection or Fontan completion: quantification with MRI. Circ Cardiovasc Imaging 2009; 2: 219-225

366 Kastler B. [Value of MRI in the evaluation of congenital anomalies of the heart and great vessels]. J Radiol 2004; 85: 1851-1853

367 McLaren CA, Elliott MJ, Roebuck DJ. Vascular compression of the airway in children. Paediatr Respir Rev 2008; 9: 85-94

368 Nielsen JC, Powell AJ, Gauvreau K et al. Magnetic resonance imaging predictors of coarctation severity. Circulation 2005; 111: 622-628

369 Prakash A, Torres AJ, Printz BF et al. Usefulness of magnetic resonance angiography in the evaluation of complex congenital heart disease in newborns and infants. Am J Cardiol 2007; 100: 715 - 721

370 Prasad SK, Soukias N, Hornung T et al. Role of magnetic resonance angiography in the diagnosis of major aortopulmonary collateral arteries and partial anomalous pulmonary venous drainage. Circulation 2004; 109: 207 - 214

371 Steffens JC, Bourne MW, Sakuma $H$ et al. Quantification of collateral blood flow in coarctation of the aorta by velocity encoded cine magnetic resonance imaging. Circulation 1994; 90: 937-943

372 Boxt LM. Magnetic resonance and computed tomographic evaluation of congenital heart disease. J Magn Reson Imaging 2004; 19: 827 847

373 Chandran A, Fricker FJ, Schowengerdt KO et al. An institutional review of the value of computed tomographic angiography in the diagnosis of congenital cardiac malformations. Cardiol Young 2005; 15: 47 - 51

374 Taylor AM, Dymarkowski S, Hamaekers P et al. MR coronary angiography and late-enhancement myocardial MR in children who underwent arterial switch surgery for transposition of great arteries. Radiology 2005; 234: 542 - 547

375 Arnold R, Ley S, Ley-Zaporozhan J et al. Visualization of coronary arteries in patients after childhood Kawasaki syndrome: value of multidetector CT and MR imaging in comparison to conventional coronary catheterization. Pediatr Radiol 2007; 37: 998 -1006

376 Hong C, Woodard PK, Bae KT. Congenital coronary artery anomaly demonstrated by three dimensional 16 slice spiral CT angiography. Heart 2004; 90: 478

377 Turner A, Gavel G, Coutts J. Vascular rings - presentation, investigation and outcome. Eur J Pediatr 2005; 164: 266-270

378 Caputo GR, Kondo C, Masui T et al. Right and left lung perfusion: in vitro and in vivo validation with oblique-angle, velocity-encoded cine MR imaging. Radiology 1991; 180: 693-698

379 Fratz S, Hess J, Schwaiger M et al. More accurate quantification of pulmonary blood flow by magnetic resonance imaging than by lung perfusion scintigraphy in patients with fontan circulation. Circulation 2002; 106: $1510-1513$

380 Klimes K, Abdul-Khaliq H, Ovroutski S et al. Pulmonary and caval blood flow patterns in patients with intracardiac and extracardiac Fontan: a magnetic resonance study. Clin Res Cardiol 2007; 96: 160-167

381 Brenner LD, Caputo GR, Mostbeck G et al. Quantification of left to right atrial shunts with velocity-encoded cine nuclear magnetic resonance imaging. J Am Coll Cardiol 1992; 20: 1246-1250

382 Hundley WG, Li HF, Lange RA et al. Assessment of left-to-right intracardiac shunting by velocity-encoded, phase-difference magnetic resonance imaging. A comparison with oximetric and indicator dilution techniques. Circulation 1995; 91: 2955-2960

383 Beerbaum P, Barth P, Kropf S et al. Cardiac function by MRI in congenital heart disease: impact of consensus training on interinstitutional variance. J Magn Reson Imaging 2009; 30: 956-966

384 Fratz S, Schuhbaeck A, Buchner C et al. Comparison of accuracy of axial slices versus short-axis slices for measuring ventricular volumes by cardiac magnetic resonance in patients with corrected tetralogy of fallot. Am J Cardiol 2009; 103: $1764-1769$ 
385 Zahn EM, Hellenbrand WE, Lock JE et al. Implantation of the melody transcatheter pulmonary valve in patients with a dysfunctional right ventricular outflow tract conduit early results from the u.s. Clinical trial. J Am Coll Cardiol 2009; 54: 1722-1729

386 Buechel ER, Balmer C, Bauersfeld U et al. Feasibility of perfusion cardiovascular magnetic resonance in paediatric patients. J Cardiovasc Magn Reson 2009; 11: 51

387 Gutberlet M, Boeckel T, Hosten N et al. Arterial switch procedure for Dtransposition of the great arteries: quantitative midterm evaluation of hemodynamic changes with cine MR imaging and phase-shift velocity mapping-initial experience. Radiology 2000; 214: 467-475

388 Sakuma H, Ichikawa Y, Chino S et al. Detection of coronary artery stenosis with whole-heart coronary magnetic resonance angiography. J Am Coll Cardiol 2006; 48: 1946-1950

389 Gutberlet M, Hoffmann J, Kunzel E et al. [Preoperative and postoperative imaging in patients with transposition of the great arteries]. Radiologe 2011; 51: 15-22

390 Cohen MD, Johnson T, Ramrakhiani S. MRI of surgical repair of transposition of the great vessels. Am J Roentgenol 2010; 194: 250-260

391 Fogel MA, Hubbard A, Weinberg PM. A simplified approach for assessment of intracardiac baffles and extracardiac conduits in congenital heart surgery with two- and three-dimensional magnetic resonance imaging. Am Heart J 2001; 142: 1028 - 1036

392 Hager A, Kaemmerer H, Leppert A et al. Follow-up of adults with coarctation of the aorta: comparison of helical CT and MRI, and impact on assessing diameter changes. Chest 2004; 126: 1169-1176

393 Krishnam MS, Tomasian A, Deshpande V et al. Noncontrast 3D steadystate free-precession magnetic resonance angiography of the whole chest using nonselective radiofrequency excitation over a large field of view: comparison with single-phase 3D contrast-enhanced magnetic resonance angiography. Invest Radiol 2008; 43: 411-420

394 Masui T, Katayama M, Kobayashi S et al. Gadolinium-enhanced MR angiography in the evaluation of congenital cardiovascular disease pre- and postoperative states in infants and children. J Magn Reson Imaging 2000; 12: $1034-1042$

395 Potthast S, Mitsumori L, Stanescu LA et al. Measuring aortic diameter with different MR techniques: comparison of three-dimensional (3D) navigated steady-state free-precession (SSFP), 3D contrast-enhanced magnetic resonance angiography (CE-MRA), 2D T2 black blood, and 2D cine SSFP. J Magn Reson Imaging 2010; 31: 177-184 